\title{
Wie aus Umweltforschung die genetische Pränataldiagnostik entstand
}

\author{
Über eine Methodenverschiebung in der Vorsorge um 1970
}

\author{
Birgit Nemec und Fabian Zimmer
}

The Emergence of Genetic Prenatal Diagnosis from Environmental Research. On a Methodological Shift in Prevention Around 1970

\begin{abstract}
The history of genetic prenatal diagnosis has so far been analyzed as a part of the history of human genetics and its reorientation as a clinical and laboratory-based scientific discipline in the second half of the 20th century. Based on new source material, we show in this paper that the interest in prenatal diagnosis also arose within the context of research on mutagenicity (the capacity to induce mutations) that was concerned with environmental dangers to human health. Our analysis of the debates around the establishment of the German Research Foundation's (DFG) research program "Prenatal Diagnosis of Genetic Defects" reveals that amniocentesis was introduced in Western Germany by a group of scientists working on the dangers for the human organism caused by radiation, pharmaceuticals, and other substances and consumer goods. We argue that, in a period of growing environmental concern, the support of prenatal diagnosis aimed to close a perceived gap in the prevention of environmental mutagenicity, i. e. genetic anomalies induced by environmental factors. The expected financing of prenatal diagnosis by health insurance in the course of the reform of abortion rights was used as another argument for the new technology's introduction as a "defensive measure". Only in a second step did changes in research structures, but most importantly experience from gynecological practice lead to a reframing of the technology as a tool for the diagnosis and prevention of mostly genetic or spontaneously occurring anomalies. Eventually, prenatal diagnosis, as it became routinely used in Western Germany from the early 1980s onward, had little to do with "environmental" questions. This case study of the early history of genetic prenatal diagnosis analyzes the still poorly researched relationship between research in human genetics, environmental research and medical practice. Furthermore, we aim to shed new light on a shift in perspective in prevention around 1970 that has so far been described in different contexts.
\end{abstract}

Die Geschichte der genetischen Pränataldiagnostik ist bislang als Teil der Geschichte der Humangenetik und deren Neuorientierung als klinisch-laborwissenschaftliche Disziplin in der zweiten Hälfte des 20. Jahrhunderts betrachtet worden. Anhand neuen Quellenmaterials soll in diesem Beitrag gezeigt werden, dass das Interesse an der Pränataldiagnostik in Westdeutschland auch im Kontext von Forschungen entstand, die sich mit Gefahren für den Menschen in der Umwelt befassten. Anhand der Debatten um die Einrichtung des DFG-Schwerpunktprogramms „Pränatale Diagnostik genetischer Defekte” 1970 untersuchen wir, wie die Technik der Amniozentese in Westdeutschland von einer interdisziplinären Forschungsgruppe eingeführt wurde, die sich mit Schädigungen des Organismus durch Strahlen, Arzneimittel und andere Gebrauchsstoffe und Konsumgüter befasste. In einer als ökologische Wende bezeichneten Zeit wachsenden Umweltbewusstseins, so unsere These, sollte durch die Förderung der Pränataldiagnostik eine wahrgenommene Lücke in der Prävention umweltbedingt auftretender genetischer Anomalien geschlossen werden. Für die Pränataldiagnostik als "Schutzmaßnahme" sprach unter anderem ihre erwartete Finanzierung als Krankenkassenleistung im Zuge der Reform des Abtreibungsrechts. Erst in einem zweiten Schritt führten veränderungen von Strukturen der Forschung, vor allem aber Erfahrungen in der gynäkologischen Praxis zu einer Neuausrichtung auf die Diagnostik und Prävention mehrheitlich erblicher oder spontan auftretender Anomalien. Die Pränataldiagnostik, so wie sie schließlich in Westdeutsch- 
land ab den frühen 1980er Jahren routinemäßig Einsatz fand, hatte mit Fragen der "Umwelt" kaum noch zu tun. Diese Fallstudie zur Frühgeschichte der genetischen Pränataldiagnostik handelt von dem noch wenig untersuchten Verhältnis von humangenetischer Forschung, klinischer Praxis und Umweltforschung und hat zum weiteren Ziel, den bisher in anderen Kontexten beschriebenen Wandel von Perspektiven in der Vorsorge um 1970 zu beleuchten.

\section{Einleitung}

Im April 1970 erfuhr das Ehepaar Knörr - sie, Henriette Knörr-Gärtner (1916-1998), war Leiterin der Sektion Zytogenetik an der Ulmer Universitätsfrauenklinik; er, Karl Knörr (1915-1996), war Professor für Gynäkologie und Geburtshilfe ebenfalls in Ulm - auf dem 5. Weltkongress der Gynäkologie und Geburtshilfe in New York von einem medizinischen Verfahren, das dem Bereich der Zytogenetik ein praktisches Anwendungsgebiet eröffnete: die Technik der Amniozentese. Mit Verfahren der Zellentnahme und -kultivierung war bereits seit einigen Jahren in Deutschland und den USA experimentiert worden. Bei einem anschließenden Besuch im Johns Hopkins Hospital in Baltimore ließ sich das Ehepaar Knörr, oder die „Knörrs“, wie sie unter Kollegen bekannt waren (Knörr-Gärtner 1995: 317), über die technischen Details einer neuen Form des Eingriffes instruieren, der den Einsatz der Zellentnahme bei fortbestehender Schwangerschaft erlaubte - als Form der pränatalen Diagnose eventueller Chromosomen- oder Stoffwechselanomalien. Durch Punktion mit einer langen Nadel entnahm man Fruchtwasser aus der Gebärmutter der Schwangeren und führte an den im Fruchtwasser enthaltenen Zellen des Fötus, nachdem man sie im Labor kultiviert hatte, zytogenetische und biochemische Untersuchungen durch. Mit „Überzeugung und Freude“, wie sie in autobiographischen Notizen zu Protokoll gaben (ebd.: 316), erprobten die Knörrs nach ihrer Rückkehr nach Deutschland die Technik erfolgreich in der eigenen Klinik und im eigenen Labor. Karl Knörr publizierte 1971 ein erstes deutschsprachiges Paper: „Möglichkeiten und Konsequenzen der pränatalen Diagnostik kongenitaler Anomalien“ (Knörr 1971; vgl. Knörr 1995; Knörr-Gärtner 1995; Knörr et al. 1995). In den folgenden Jahren erfuhr die Technologie in Westdeutschland eine rasche Etablierung dank maßgeblicher Förderung durch die Deutsche Forschungsgemeinschaft (DFG). Im Rahmen des Schwerpunktprogramms (SP) „Pränatale Diagnostik genetisch bedingter Defekte“ (1973-1979) wurde das Verfahren an einer wachsenden Zahl von Standorten in Westdeutschland eingeführt und so umfangreich medizinisch-technisch weiterentwickelt, dass es nach Ablauf des Schwer- 
punktprogramms als pränataldiagnostische Routineleistung, nunmehr von den Krankenkassen getragen, die medizinische Praxis und die Erfahrung von Schwangerschaft für Millionen Frauen und Familien nachhaltig prägen sollte.

Dieser Beitrag beleuchtet die Frühgeschichte der Etablierung der genetischen pränatalen Diagnostik in Westdeutschland und damit ein bislang noch wenig erforschtes Thema. Unser besonderes Interesse gilt dabei einem an dieser Fallstudie zu beobachtenden Wandel in der Prävention von Umweltmutagenität, also dem umweltbedingten Auftreten genetischer Anomalien, und damit einem Wandel des Verhältnisses von humangenetischer Forschung, klinischer Praxis und Umweltforschung. Bisherige Studien haben die genetische Diagnostik als Teil der Geschichte der Humangenetik betrachtet und ihre Implementierung in die Geschichte der Neuorientierung der Disziplin durch genuin naturwissenschaftlich-medizinische Methoden der Geschichte der Chromosomengenetik sowie in Tendenzen der Individualisierung medizinischer Angebote in der zweiten Hälfte des 20. Jahrhunderts eingeordnet (Thomaschke 2014; Waldschmidt 1996; Nippert 1991; Cottebrune 2008: 223-235, Cottebrune 2015; De Chadarevian 2018). Auch wurde für einen internationalen Kontext gezeigt, dass die Generalisierung der Pränataldiagnostik in den 1980er Jahren mit der Neudefinition von Down-Syndrom als einem Problem der öffentlichen Gesundheit in Zusammenhang stand (Löwy 2017; Löwy 2014a). Unser Beitrag setzt demgegenüber bei der zunächst überraschenden Beobachtung an, dass das Interesse an der genetischen Pränataldiagnostik in Westdeutschland auch im Kontext von Forschungen entstand, die sich mit der Prävention von exogen ausgelösten Mutationen befassten, also mit Schädigungen des menschlichen Erbguts durch Stoffe in der Umwelt. Neben Strahlen lösten Arzneimittel, aber auch andere Gebrauchsstoffe und Konsumgüter als vermutete Gefahren für das Erbgut der gegenwärtigen und folgenden Generationen eine Suche nach Instrumenten zur Regulierung dieser schädlichen Umwelteinflüsse aus. Unsere Analyse dieser Suche nach effektiven Formen der Prävention von Umweltmutagenität nimmt die Zeit um 1970 in den Fokus und basiert im Besonderen auf Akten des erwähnten DFG-Schwerpunktprogramms „Pränatale Diagnostik genetisch bedingter Defekte“, einem Unterbestand der Akten der sogenannten DFG-Kommission für Mutagenitätsfragen, einer staatsnahen Einrichtung zur Kontrolle von Mutagenität. In den Monaten rund um die USA-Reise der Knörrs wurde ein Interesse an der genetischen Pränataldiagnostik in unterschiedlichen Forschungszusammenhängen geäußert. Wie die umfangreiche Dokumentation der Vorgespräche der am späteren Schwerpunktprogramm beteiligten Wissenschaftler*innen unterschiedlicher Disziplinen zeigt, erfolgte die Förderung der Pränataldiagnostik jedoch ganz explizit, um eine 
wahrgenommene Lücke in bisherigen Formen der Regulierung von Umweltmutagenität zu schließen. Der Anstoß für dessen Förderung sowie die organisatorische Leitung des Programms zwischen 1973 und 1979 erfolgte daher durch die Kommission für Mutagenitätsfragen. Erst in einem späteren Schritt verschob sich der Einsatzbereich der Amniozentese zum heute noch gültigen Fokus auf die Früherkennung spontan auftretender Anomalien (etwa Down-Syndrom) oder in der Familie bekannter erblicher Erkrankungen. Im Zentrum dieses Beitrags steht die Chronologie dieser Methodenverschiebung in der Vorsorge um 1970: der Entstehung der genetischen Pränataldiagnostik aus der Mutagenitätsforschung.

Im ersten Teil des Beitrags untersuchen wir, wie Gefahren und Unsicherheiten des „Atomzeitalters“ für die Gesundheit von Mensch und Natur sowie medizinische Skandale, wie die Contergan-Fehlbildungen, Umweltfragen im Laufe der 1950er und 1960er Jahre zum dringlichen Thema medizinischer und biologischer Forschungsinitiativen machten, von denen man sich eine wissenschaftliche Grundlage für politische Regulierungsbestrebungen erhoffte. Auch Karl Knörr und Henriette Knörr-Gärtner forschten vor ihrer Rolle als Pioniere der Amniozentese in Westdeutschland zur Prävention von Umweltnoxen, speziell in der Strahlenforschung und der Teratologie. Viele dieser Bestrebungen mündeten um 1970 in der in der Umweltgeschichte als „ökologische Wende“ bezeichneten Herausbildung eines globalen Umweltbewusstseins (Radkau 2011) und bewirkten die Schaffung zahlreicher dem Umweltschutz verschriebener Initiativen und Institutionen, die der Prävention einer drohenden „Umweltkrise“ dienen sollten. „Umwelt“ wurde in dieser Phase als ein durch „zunehmende Umweltbelastungen“ gekennzeichneter "menschlicher Lebensraum“ (DFG 1971a: 10) konzipiert, und galt folglich als eine wichtige „Aufgabe für Forschung und praktische Politik“ (ebd.).

Wir zeigen im zweiten Teil dieses Beitrags, wie diese ökologische Perspektive in der Frühphase der Begründung der Förderung der Pränataldiagnostik aufgegriffen wurde, indem diese als „erweitertes“ Aufgabengebiet der Mutagenitätsforschung zur Prävention einer erwarteten Verschlechterung des Erbguts dringend empfohlen wurde. In den ersten Wochen waren diese Überlegungen von Ansätzen einer ökologisch orientierten Genetik geprägt, wie sie die Kommission für Mutagenitätsfragen bisher gefördert hatte. Im Lauf der Arbeitsgespräche der folgenden Monate verbanden sich diese umwelthygienischen Überlegungen allerdings mit Argumenten aus dem Bereich der medizinischen Praxis und Gesundheitspolitik. Dieser Schritt rückte, parallel zum Verblassen von Vorstellungen staatlich gesteuerter, technokratischer Gefahrenregulierung, das Individuum als Interventionsobjekt in den Fokus der Vorstellungen von genetischer Vorsorge. Speziell die Aussicht auf die Reform des Abtreibungsparagraphen 218 StGB 
(sie erfolgte letztlich 1976) und auf die Aufnahme der genetischen Pränataldiagnostik in den Leistungskatalog der Krankenkassen als sogenannte flankierende Maßnahme bot den beteiligten Forschern eine vielversprechende Perspektive zur Finanzierung ihrer Interessen. Erst in der anschließenden praktischen Umsetzung in der gynäkologischen klinischen Praxis wurde der Fokus im Einsatz der Amniozentese auf die Reduktion erblicher, vor allem aber spontan auftretender Anomalien gelegt.

Ziel unseres Beitrags ist es, diesen Schritt von einem Forschungsinteresse für Umweltnoxen $\mathrm{zu}$ einem auf individuelle oder erbliche Risiken ausgerichteten klinisch-praktischen Verfahren in seinen medizin- und wissenschaftsgeschichtlichen Zusammenhängen darzustellen. Diese Fallstudie zur Frühgeschichte der genetischen Pränataldiagnostik handelt damit von dem noch wenig erforschten Verhältnis von Humangenetik, medizinischer Klinik und Umweltforschung und hat zum weiteren Ziel, den in anderen Kontexten beschriebenen Wandel von Perspektiven in der Vorsorge um $1970 \mathrm{zu}$ beleuchten.

\section{Gefahren für das Erbgut und deren Prävention vor 1970}

„Muß der technische Fortschritt mit dem genetischen Tod bezahlt werden?" So zugespitzt präsentierte DFG-Präsident Julius Speer 1969 in seiner Rede zur Eröffnung des „Zentrallaboratoriums für Mutagenitätsprüfung“ (ZLM) in Freiburg im Breisgau die leitende Forschungsfrage zahlreicher Institutionen der DFG. Diese Frage habe den ursprünglichen Impuls zur Gründung der Kommission für Mutagenitätsfragen im Jahre 1964 und zur Einrichtung des von der Kommission entworfenen und koordinierten Labors fünf Jahre später gegeben (DFG 1970: 18). Unter „Mutagenen“ verstand man Stoffe oder Strahlen, die das menschliche Erbgut verändern konnten. Diese Veränderungen waren irreversibel und konnten, wenn die Keimzellen betroffen waren, weitervererbt werden. Genetiker warnten daher vor einem Szenario, in dem „Evolution“ - oder wie man es immer nennen sollte - eine Richtung einschlägt, die für die Menschheit katastrophal werden würde“, wie es ein Mitglied der Kommission für Mutagenitätsfragen bei der auf Speers Rede folgenden Pressekonferenz abschließend zusammenfasste (ebd.: 39). Das ZLM sollte mit Routinemutagenitätstests an einer Vielzahl von Substanzen einen Beitrag dazu leisten, das Eintreten dieses Szenarios zu verhindern.

Die wissenschaftliche Beschäftigung mit den Gefahren der technisierten Umwelt war, wie auch Speer selbst in seiner Eröffnungsrede betonte, für die DFG keineswegs neu (vgl. ebd.: 18). Die DFG förderte seit ihrer 
Neugründung nach dem Krieg großangelegte Forschungsprojekte, die der wissenschaftlich fundierten Regulierung solcher Gefahren dienen sollten und sich als Brücke zwischen Grundlagen- und Anwendungswissenschaften verstanden (vgl. Kaldewey \& Schauz 2018). Der Senat, der 1951 mit der neuen Satzung der DFG geschaffen wurde und mit hochrangigen Wissenschaftlern, Politikern und Vertretern aus der Industrie besetzt war, konnte sogenannte Senatskommissionen berufen, deren Zweck die wissenschaftliche Beratung der Regierung war und die hierfür Forschungsprojekte anregen konnten. Hierzu zählte insbesondere das neue Förderinstrument der Schwerpunktprogramme, das ab Mitte der 1950er Jahre neben die bis dahin übliche Einzelförderung im Normalverfahren trat. Außer der Zuarbeit für die Kommissionen sollten mit solchen Großforschungsprojekten wahrgenommene Lücken in der westdeutschen Forschungslandschaft geschlossen werden - Themen gründlich und koordiniert in einer Art bearbeitet werden, wie es im Normalverfahren nicht zu verwirklichen war (zum Senat und seinen Kommissionen und Aufgaben allgemein vgl. Orth 2011: 51-54; 125-149).

$\mathrm{Zu}$ diesen Kommissionen gehörten zwar auch einige geistes-, sozialoder ingenieurswissenschaftliche Gruppen, ein ganz überwiegender Teil der Kommissionen aber befasste sich, wie die jüngste Forschung zur Geschichte der DFG gezeigt hat, mit umweltbedingten Gefahren für den menschlichen Organismus in einer zunehmend technisierten Welt und ihrer Regulierung: eine Kommission zur Prüfung fremder Stoffe bei Lebensmitteln, eine Farbstoff-Kommission, eine Kommission für Lärmforschung, eine Kommission zur Erforschung der Luftverunreinigung, eine für Lebensmittelkonservierung, eine für Berufskrebs und viele mehr, die sämtlich im Laufe der 1950er und 1960er Jahre eingerichtet worden waren (vgl. ebd.; Schwerin 2010a; Schwerin 2014; Schwerin 2015: 308-315; Moser 2011: 250-252; Stoff 2012: 309-322; Bächi 2010). Diese Senatskommissionen stellten eine Schnittstelle zwischen Forschung und einer technokratisch konzipierten Regulierung von Gefahren dar (Orth 2011: 51-54; 125-149). Sie waren teilweise untereinander personell eng verknüpft und ihr $\mathrm{Zu}$ standekommen und ihre Arbeit war im Speziellen durch zwei Themen geprägt: Zum einen hatten Genetiker bereits seit den 1950er Jahren wiederholt, auch öffentlich, vor einem Anstieg der natürlichen Mutationsrate gewarnt, der durch radioaktiven Fallout, die Proliferation von Radioisotopen und durch die in der entstehenden Konsumgesellschaft rasant zunehmenden Zahl neuartiger Stoffe in Lebensmitteln, Verpackungen, Kleidung etc. verursacht werden konnte (vgl. Schwerin 2009; Schwerin 2012a). Zum anderen hatte das Bekanntwerden schwerer Schädigungen beim ungeborenen Kind durch die Einnahme des gebräuchlichen Schlafmittels Contergan während der Schwangerschaft Anfang der 1960er Jahre die Wahrnehmung 
einer „toxischen Gesamtsituation“ (Stoff 2009; zur Gefahrstoffproblematik allgemein Stoff 2015) verstärkt und Forschungsförderung zur Sicherung der Gesundheit insbesondere des ungeborenen Kindes und damit darauffolgender Generationen als umso dringlicher erscheinen lassen.

Der Untersuchungszeitraum der bisherigen Studien zu diesen Kommissionen endet um 1970, so dass die hier beleuchteten Entwicklungen, aus denen heraus die Entscheidung zur Förderung der Pränataldiagnostik fiel, bisher noch nicht genauer betrachtet worden sind. In diesem Kapitel soll also einerseits gezeigt werden, wie sich im Rahmen der auf die politische Regulierung von exogenen Gefahren für das Erbgut abzielenden DFGForschungspolitik der 1960er Jahre eine enge Zusammenarbeit von Forscher*innen und Kliniker*innen, ${ }^{1}$ speziell in den Bereichen Gynäkologie und Humangenetik, entwickelte, und wie diese sowohl institutionell wie auch rhetorisch als „Umweltforschung“ begriffen wurde, mit der die Erhaltung der menschlichen Lebensgrundlagen in einer technisieren Umwelt gesichert werden sollte. Wie sich die Auffassung von genetischer Prävention in dieser Phase konkret veränderte, zeigen wir zudem exemplarisch anhand der Biographien des Ehepaars Knörr und deren Arbeit in Strahlenforschung, Teratologie und Mutagenitätsprüfung.

\section{Das Ehepaar Knörr in Strahlenforschung und Teratologie}

Als in den 1950er Jahren die Angst vor Strahlenschäden durch atmosphärische Atombombenversuche und radioaktiven Fallout zu einem zentralen Thema der öffentlichen Debatten wurde, erhielten wissenschaftliche Fragen nach Sicherung von Gesundheit und Erbgut des Menschen zunehmend Unterstützung. Wie die bisherige Forschung gezeigt hat, konnte sich die durch ihre Nähe zur nationalsozialistischen Rassenhygiene diskreditierte Vererbungslehre durch Förderung der DFG, aber auch anderer Institutionen wie der Deutschen Atomkommission, als moderne, international und an molekularbiologischen Methoden ausgerichtete Humangenetik neu etablieren und alte Degenerationsängste in einer veränderten Gefahrensituation neu artikulieren (vgl. Cottebrune 2008: Kap. 4; Kröner 1997: 32-35; Weingart et al. 1988: 630-668; Schwerin 2010b; Schwerin 2012a). ${ }^{2}$

Auch die wissenschaftliche Karriere Henriette Knörr-Gärtners begann im Kontext strahlenbiologischer Fragen. Nach Studium und Promotion in Medizin in der unmittelbaren Nachkriegszeit bei dem Gynäkologen und Geburtshelfer Werner Bickenbach (1900-1974) hatte sie sich mit Forschungen zur gynäkologischen Strahlentherapie habilitiert. Als Mitarbeiterin des Arbeitskreises für Strahlenbiologie der Deutschen Atomkommission hatte sie, wie sie in einem autobiographischen Rückblick schreibt, „die Aufdeckung des menschlichen Karyotypus“ (Knörr-Gärtner 1995: 315) durch die Methoden der Zytogenetik miterlebt. Sie beschrieb diese Entde- 
ckung als dramatische Horizonterweiterung der Forschungen zur Gefährdung des Menschen durch seine radioaktive Umwelt: „Aus dem im Rahmen der Strahlentherapie des einzelnen Kranken zunächst irrelevanten Problem der genetischen Strahlenbelastung war im Atomzeitalter ein überindividuelles weltweites Anliegen geworden [...]" (ebd.). Die Karyogramme, die die menschlichen Chromosomen und ihre Fehlbildungen erstmals sichtbar machten, interpretierten die Forscher vor dem Hintergrund des atomaren Ost-West-Konflikts als „Kartographien menschlicher Verwundbarkeit in einer von technischen Bedrohungen geprägten Welt", wie Philipp Osten es pointiert formuliert hat (Osten 2012: 157; vgl. auch Schwerin 2009). Für Henriette Knörr-Gärtner, wie für viele andere Forscher*innen auf dem Gebiet der Strahlenbiologie, „bedeuteten diese Erkenntnisse den Einstieg in die Humangenetik“ (Knörr-Gärtner 1995: 315; vgl. für weitere Fälle etwa Schwerin 2012b: 109). Sie sorgte gemeinsam mit ihrem Mann im Laufe der 1960er Jahre zunächst in Tübingen, dann ab 1967 in Ulm für die Bereitstellung von Räumlichkeiten und Infrastrukturen für die Zytogenetik und förderte deren Expertise. Ab 1968 leitete sie die Sektion Zytogenetik der Ulmer Universitätsklinik und leistete hier, wie sie im Rückblick meinte, „eine gewisse Pionierarbeit auf dem Gebiet der Pränatalen Diagnostik“, dank der ihr Labor dann auch die Ausbildung von Mitarbeiter*innen der an dem DFG-Schwerpunktprogramm beteiligten Institute übernehmen konnte (Knörr-Gärtner 1995: 316).

Auch Karl Knörr arbeitete seit den frühen 1960er Jahren an zytogenetischen Chromosomenuntersuchungen. Wie seine spätere Frau war auch Knörr ein Schüler Bickenbachs und studierte bei ihm Gynäkologie und Geburtshilfe zunächst mit dem Ziel, Hausarzt zu werden. Auf Anregung Bickenbachs, den beide Knörrs in ihren autobiographischen Notizen als ihren Mentor priesen, entwickelte er aber ein wissenschaftliches Interesse an der Genetik und der Teratologie, also der "Missbildungsforschung“ (vgl. Knörr 1995: 307 f.). Als frisch ernannter Professor mit Spezialisierung auf diesem Gebiet gehörte er ab 1963 der DFG-Kommission für teratologische Fragen an. Diese Kommission war im selben Jahr unter dem Eindruck der Contergan-Fehlbildungen gegründet worden, mit dem Ziel ein teratologisches Schwerpunktprogramm zu konzipieren. Schon im folgenden Jahr nahm ein solches Programm unter dem Titel „Mißbildungsentstehung und Mißbildungshäufigkeit" die Arbeit auf (vgl. Cottebrune 2008: 223-235). Mit dem Programm sollte der „wissenschaftlich unerträglich[e]“ Umstand behoben werden, „daß kein gesichertes Wissen darüber vorlag, ob irgendwelche Einflußfaktoren, denen eine Schwangere in ihrem täglichen Leben ausgesetzt ist und die als vielleicht harmlos angesehen werden, ernste Gefahren für den Verlauf der Schwangerschaft und der Entwicklung des Kindes bewirken könnten“, wie der Leiter der Kommission, der 


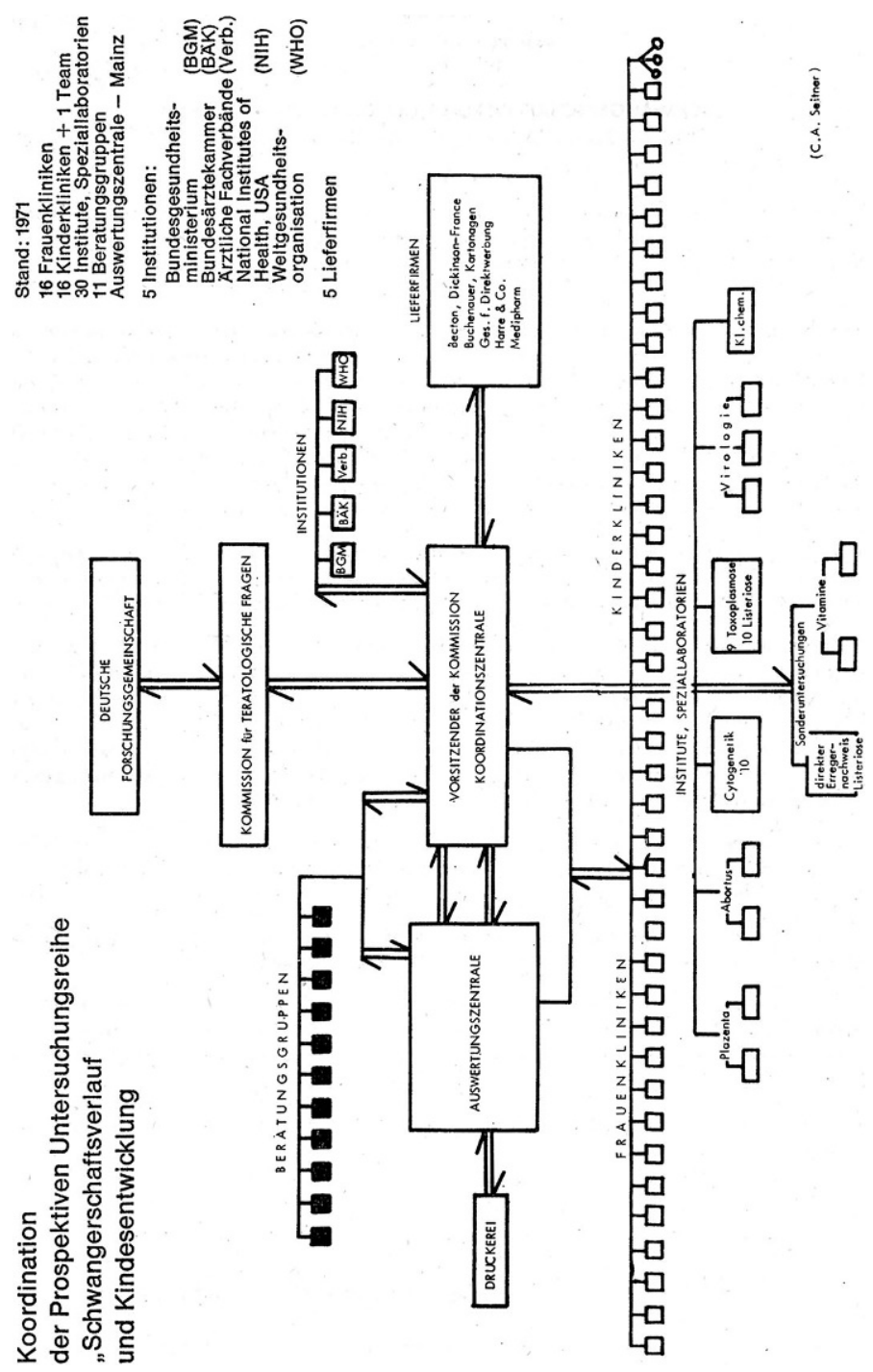

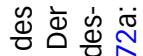

产电亭

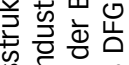

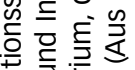

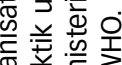

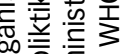

ठ응

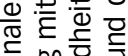

항ㅎㅎ

잉

至

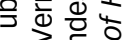

㕸

O

㟧

음 $\frac{0}{3}$

t

बis

:

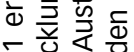

人

政

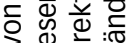

政

号.

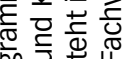

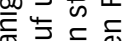

등

के

क क

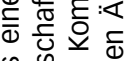

乐

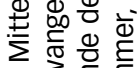

- 绕 旅

吅 离

选的产督氐

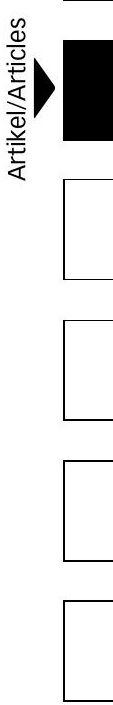


Frankfurter Genetiker Karl-Heinz Degenhardt (1920-1994), im Rückblick zusammenfasste (DFG 1977a: 9). Von 1964 bis 1977 mit insgesamt über 20 Millionen DM von der DFG gefördert, wurden in dem Schwerpunktprogramm über 14.000 Schwangerschaften und daraus resultierende Kinder, Totgeburten, Aborte (und Plazenten) in einem engmaschigen Monitoring überwacht: Ess- und Trinkverhalten der Mutter, sozio-ökonomische Faktoren, Arbeitsalltag, Medikamentenkonsum, Vitaminzufuhr, Krankheiten, körperliche Betätigungen wie Gartenarbeit und vieles mehr - etwa die Haltung von Wellensittichen im Haushalt - wurden registriert und mit Beobachtungsdaten zur Entwicklung des Kindes sowie mit pathologischen Befunden in Bezug gesetzt (Koller 1983). Karl Knörr entwickelte die Fragebögen, mit denen die Datenerhebung bei den Müttern durchgeführt wurde. Im Laufe des Programms war er dann für seinen engeren Fachbereich zuständig und arbeitete ebenfalls mit zytogenetischen Methoden (vgl. DFG 1965: 277; DFG 1966: 313), die dazu dienen sollten, erbliche Faktoren von Umwelteinflüssen abzugrenzen. Hiermit erweiterte sich bereits das Spektrum des Schwerpunktprogramms über die zunächst rein statistische Methodik hinaus. Um dieser zunehmend breiten Ausrichtung des Programms Rechnung zu tragen, wurde dies bereits 1966 in „Schwangerschaftsverlauf und Kindesentwicklung" umbenannt.

Wie die Medizinhistorikerin Anne Cottebrune herausgestellt hat, zeichnete sich dieses erste großangelegte Programm Westdeutschlands zur Erforschung von Umwelteinflüssen auf die Entwicklung des Kindes insgesamt durch eine sehr breite Interdisziplinarität aus (vgl. Cottebrune 2008: 231-235). Auch wenn der Vorsitz der Kommission und die Aufsicht über das Programm von Karl-Heinz Degenhardt und Friedrich Vogel (1925-2006), zwei renommierten Humangenetikern mit Interesse für die Zytogenetik, geführt wurden, so vereinte das Programm doch die zahlreichen verschiedenen am un- bzw. neugeborenen Kind interessierten Disziplinen und zugleich Klinik und Forschung, indem es Gynäkologen, wie Karl Knörr, erstmals in enge Zusammenarbeit mit Frauen- und Kinderheilkundlern, mit Orthopäden, Pathologen sowie mit Zytogenetikern und Statistikern brachte. Diese neue Organisationsstruktur lässt sich anhand einer Visualisierung des Schwerpunktprogramms besonders eindrücklich verfolgen (vgl. Abb. 1). Ganz im Sinne der risikopolitischen Ausrichtung der Senatskommissionen sollte im Falle der Entdeckung weiterer schädlicher Einflussfaktoren ein direkter Kontakt mit relevanten Regierungsstellen, Standesvertretungen und internationalen Organisationen wie der WHO hergestellt werden, um Schritte für die politische Regulierung und Beseitigung dieser Gefahren einzuleiten (Ebd.). 


\section{Die Mutagenitätsforschung der DFG}

Ein dritter Bereich, neben Strahlenforschung und Teratologie, in dem in den 1960er Jahren zur Prävention von exogenen Schädigungen des Menschen und speziell des Erbguts - zunächst ohne die aktive Beteiligung der Knörrs - gearbeitet wurde, war die Mutagenitätsforschung. Im Jahr 1963, ebenfalls unter dem Eindruck der Contergan-Schädigungen, hatte die seit 1949 bestehende Farbstoff-Kommission der DFG begonnen, sich mit der „Prüfung von Lebensmittelzusätzen auf etwaige mutagene oder teratogene Wirkungen“ zu beschäftigen und angeregt, zur gründlichen Bearbeitung dieser Themen eine eigene Arbeitsgruppe zu bilden, aus der im November 1964 die Kommission für Mutagenitätsfragen entstand (DFG 1964: 25 f.; vgl. Schwerin 2010a; Schwerin 2012b; Schwerin 2014). ${ }^{3}$ Auch diese Kommission war interdisziplinär aufgestellt - sie versammelte Vertreter aus Botanik, Bakteriologie, Molekular- und Humangenetik; doch auch hier hatte ein Humangenetiker den Vorsitz inne: Helmut Baitsch (1921-2007), der sich als Direktor des Freiburger Instituts für Anthropologie und $\mathrm{Hu}-$ mangenetik in diesen Jahren für eine Zusammenführung der traditionell anthropologischen und populationsgenetischen Ansätze mit neuen und kleinteiligen Methoden der Molekulargenetik in Forschung und medizinischer Lehre einsetzte und sich innerhalb der sich neukonstituierenden Disziplin der Humangenetik als Kritiker der NS-Eugenik positionierte (vgl. Cottebrune 2012: 44-48). In dieser Auseinandersetzung mit der NS-Eugenik hatte Baitsch schon Ende der 1950er Jahre „Mutationsprophylaxe“ als die vordringlichste Aufgabe einer zeitgemäßen Eugenik beschrieben (Baitsch 1958). Das von ihm formulierte Verständnis von „Mutationsprophylaxe“ grenzte sich nach eigenen Angaben explizit von der „ausmerzenden" Eugenik des Nationalsozialismus ab und forderte vielmehr eine technokratische Regulierungspolitik, die sich exakt mit dem forschungspolitischen Modell deckte, das die DFG parallel mit ihren Senatskommissionen vorantrieb: „[E]ine der vornehmsten Aufgaben des Staates und der Staatsmänner" sei es, „durch geeignete Abkommen, Gesetze und Schutzvorschriften" die Umweltgefahren durch Strahlenexposition oder chemische Substanzen „auf ein Minimum zu reduzieren“ (ebd.: 220).

Die für diese Aufgabe benötigte wissenschaftliche Grundlage sollte dann in dem von der Kommission Anfang der 1960er Jahre entworfenen ZLM bereitgestellt werden, von dessen Einrichtung wir bereits eingangs berichtet haben. Erklärtes Ziel des ZLM war die „Überprüfung möglichst aller chemischen Substanzen unserer Umwelt auf ihre mutagene Wirkung“ (DFG 1971b: 5). Wie der Historiker Alexander von Schwerin im Detail ausgeführt hat, waren die Erwartungen an das ZLM enorm: Sämtliche der über tausend jährlich neu auf den Markt kommenden chemischen Substanzen sollten einwandfrei auf ihre Schädlichkeit geprüft werden. Die Methoden, 
derer man sich bediente, waren vielfältig und orientierten sich an den Standards, die die Molekulargenetik in den letzten Jahren etabliert hatte. Sechs verschiedene Arbeitsgruppen sollten anhand verschiedener Testsysteme an Fruchtfliegen, Hefen, Bakterien und in zytogenetischen Verfahren an menschlichen Zellkulturen prüfen, ob bei verdächtigen Substanzen mutagene Wirkungen nachzuweisen waren (Schwerin 2010a; vgl. DFG 1970: 6). Lange debattiert wurde in der Kommission zudem, ob auch Tierversuche durchgeführt werden sollen, um Einwirkungen des Stoffwechsels auf das Mutationsgeschehen erfassen zu können. 1970 entschied man sich schließlich dafür, die Arbeitsgruppe des Heidelberger Säugetiergenetikers Gunter Röhrborn (1931-2013) in die Tätigkeiten des ZLM zu integrieren (vgl. Schwerin 2012b). Die Ergebnisse der mit diesen Methoden durchgeführten Mutagenitätsprüfung sollten dann „wissenschaftliche Grundlagen für notwendige praktische Maßnahmen von Verwaltung, Gesetzgebung und Industrie [...] schaffen" (DFG 1970: 18). Dass sich die Kommission für Mutagenitätsfragen über den konkreten Weg der Regulierung - von der Feststellung einer Gefahr zum Verbot der betreffenden Substanz etwa - selber nicht völlig im Klaren war, machten bereits kritische Rückfragen von Journalisten bei der Pressekonferenz zur Eröffnung des ZLM deutlich. Wir werden auf diese Problematik im folgenden Abschnitt dieses Kapitels zurückkommen.

„Umweltforschung” in der DFG und die Wahrnehmung einer Lücke in der Regulierung von Umweltmutagenität

Um 1970 kam es zu einer wichtigen Veränderung in den Forschungsstrukturen zur Regulierung von Umweltmutagenität. Mit den skizzierten Kommissionen für Mutagenitätsfragen bzw. teratologische Fragen, in denen auch das Ehepaar Knörr tätig war, hatte sich die DFG wie bereits angesprochen im Laufe der 1960er Jahre auf dem Gebiet der Regulierung von Umweltgefahren positioniert. Nun wurden 1970 diese beiden Kommissionen und die angeschlossenen Forschungseinrichtungen, gemeinsam mit den anderen risikopolitischen Senatskommissionen der DFG, sowohl rhetorisch als auch institutionell unter dem Begriff der „Umweltforschung“ zusammengefasst und einem Senatsausschuss für Umweltforschung unterstellt. Zum einen ging es dabei um eine inhaltliche Präzisierung bislang teils disparater Vorstellungen von „Umwelt“, die man nun als durch „zunehmende Umweltbelastungen“ gekennzeichneten „menschliche[n] Lebensraum“ (DFG 1971a: 10) sowie als wichtige „Aufgabe für Forschung und praktische Politik“ definierte (ebd.). Zum anderen hatte die Zusammenfassung forschungspolitische Hintergründe. In dem neu gebildeten Ausschuss sollten Themen, Förderpolitik und Finanzplanung der bereits bestehenden Senatskommissionen besser koordiniert werden (DFG 1971c: 37). Bei der 
Vorstellung dieses neuen Forschungsschwerpunkts bekräftigte DFG-Präsident Speer abermals die risikopolitische Forschungsagenda, die die DFG seit ihrer Neugründung in den 1950er Jahren mit den Senatskommissionen verfolgt hatte:

Durch Anwendung moderner Methoden der Zusammenarbeit zwischen den Forschern der einschlägigen Disziplinen sollte jede greifbare, für die Umweltschutzproblematik wichtige wissenschaftliche Erkenntnis der Öffentlichkeit, besonders dem Gesetzgeber, bekannt gemacht und soweit wie möglich sachlich begründete Unterlagen für notwendige politische Entscheidungen erarbeitet werden (DFG 1971a: 9).

Der Zeitpunkt für die Zusammenfassung der Tätigkeiten der Senatskommissionen unter dem Begriff der Umweltforschung fiel nicht bloß zufällig just in die Zeit um 1970. Unter dem Schlagwort der „ökologischen Wende" (Radkau 2011) sind diese Jahre von der Umweltgeschichte als Epochenschwelle in der Herausbildung eines globalen Umweltbewusstseins identifiziert worden (vgl. Hünemörder 2004; Hünemörder 2005). Tatsächlich war dieser Schritt der DFG eng verknüpft mit dem Vorstoß der Bundesregierung, die 1970 ein „Sofortprogramm für den Umweltschutz“ ausgegeben hatte und im Oktober 1971 das „Umweltprogramm der Bundesregierung" verabschiedete (Umweltprogramm 1971). Wie die umwelthistorische Forschung gezeigt hat, entstand dieses Programm aus den gleichen Sorgen um die Erhaltung der menschlichen Lebensgrundlagen in einer technisierten Umwelt heraus, die Anlass zur Gründung der DFG-Senatskommissionen gegeben hatten (vgl. Hünemörder 2004; Gassert 2013). Vor allem aber stand das Umweltprogramm der Bundesregierung in derselben Tradition der technischen Gefahrenregulierung wie die Senatskommissionen, die darauf zielte, schädliche Substanzen oder Faktoren zu identifizieren und aus dem Verkehr zu ziehen. Das Umweltprogramm hatte seine Vorläufer in Institutionen wie der VDI-Kommission zur Reinhaltung der Luft oder Initiativen zum Gewässerschutz, die nunmehr unter dem neuen Schlagwort „Umweltschutz“ gebündelt wurden, dem technokratischen Ansatz aber verhaftet blieben (vgl. Engels 2006: 276-279; Uekötter 2003: 480-485). Mit der „Umweltforschung“ vollzog die DFG nicht nur die Anpassung an die von der Bundesregierung lancierten Begrifflichkeiten. Vielmehr nahm sie mit ihren Senatskommissionen eine zentrale Beratungsposition im Umweltprogramm ein (vgl. Umweltprogramm 1971: 15); der Senatsausschuss für Umweltforschung hatte überdies die Aufgabe der „Abstimmung mit dem Gesamtprogramm der Bundesregierung“. Die einzelnen Fachkommissionen wiederum sollten der „Beratung der Legislative und Exekutive“ dienen (DFG 1971c: 37). 
1971 positionierte sich die DFG als wichtige Beratungsinstitution der Umweltpolitik - also in dem Jahr, in dem mit der USA-Reise und den anschließenden Versuchen des Ehepaars Knörr in Ulm die Anwendung der Technik der Amniozentese in der Pränataldiagnostik zu einer greifbaren Option wurde. Im selben Jahr zeigten sich allerdings auch bereits erste Defizite in der Forschung zu teratogenen und mutagenen Faktoren in der Umwelt: In den beiden Senatskommissionen stellte sich die Erkenntnis ein, dass mit den gewählten Methoden und Infrastrukturen nicht jene belastbaren Aussagen zu generieren waren, die das rechtzeitige Erkennen und Beherrschen von Umweltrisiken erlaubten. Damit stießen die Kommissionen aber auf die wachsende Ungeduld einer zunehmend kritischen Medienöffentlichkeit.

Die teratologische Studie zu „Schwangerschaftsverlauf und Kindesentwicklung“ hatte sich zum Ziel gesetzt, „die ursächlichen Zusammenhänge der Entstehung angeborener Entwicklungsstörungen des Menschen“ zu klären (DFG 1965: 35). Doch bereits bei der Präsentation erster statistisch aufbereiteter Zwischenergebnisse in der Fachöffentlichkeit 1971 zeichnete sich ab, dass die Daten der Studie „überhaupt keine letzten wissenschaftlichen Klärungen schaffen" würden (Koller 1972; vgl. DFG 1972a: 54-59). Im 1977 folgenden Zwischenbericht konnten die Forscher aus der ersten großen epidemiologischen Studie der Bundesrepublik letztlich nur allgemeine Empfehlungen generieren: die "laufende Überwachung“ der Schwangerschaft und des Kleinkindes sei anzuraten, ebenso wie der vorbeugende Verzicht auf Alkohol, Rauchen und Medikamente während der Schwangerschaft (DFG 1977a: 7) - eine für epidemiologische Studien typische Kombination nicht eindeutiger Ergebnisse und zugleich klarer Verhaltensvorschriften, wie sie etwa auch Virginia Berridge in ihrer Arbeit zu Rauchen und Gesundheitsvorsorge dargelegt hat (Berridge 2007).

Als noch grundlegender erwiesen sich allerdings die Probleme der $\mathrm{Mu}-$ tagenitätsforschung. Die angewendeten Testverfahren hatten sich als wesentlich aufwändiger herausgestellt als angenommen: Tatsächlich konnten die beteiligten Wissenschaftler im Zentrallabor bis 1973 nur 61 Substanzen prüfen, und dabei nicht einmal mit hundertprozentiger Sicherheit deren Unschädlichkeit garantieren (vgl. Schwerin 2010a: 409 f.). Unter den Kommissionsmitgliedern herrschte überdies große Uneinigkeit über die Aussagekraft der verschiedenen Testsysteme und ihre Übertragbarkeit auf den Menschen. Die Auseinandersetzung kulminierte im Januar 1971 in zwei richtungsweisenden Beschlüssen: der Integration erheblich teurerer, jedoch für den Menschen aussagekräftigerer Tests im Tierversuch an Mäusen durch die Angliederung der Arbeitsgruppe des Heidelberger Genetikers Gunter Röhrborn an das ZLM, sowie der Neubesetzung der Kommission, um die Konkurrenzsituation zwischen den Vertretern der 
verschiedenen Methoden aufzulösen (ebd.: $408 \mathrm{f}$.). Gravierender als diese methodischen Probleme erwies sich allerdings, was bereits bei der Pressekonferenz 1969 von den anwesenden Journalisten wie etwa Ekkehard Sass vom Südwestfunk kritisch angesprochen worden war: „Sind Sie irgendwie vorbereitet auf den doch sicher sehr harten Kampf mit der Industrie?" (DFG 1970: 37) Hierauf hatten die Mitglieder der Kommission nur vage Antworten parat und in den folgenden Jahren wurde die Arbeit des ZLM in der Presse kritisch kommentiert und eine Beschleunigung der Forschungen gefordert (vgl. Schwerin 2010a: 409). Eine erfolgreiche politische Durchsetzung der Mutationsprävention erwies sich also hindernisreich - zumal keine vollends zuverlässigen Ergebnisse vorlagen. Im Rückblick resümierten die Forscher, das ZLM sei in einer „optimistische(n) Phase“ entworfen worden, in der man die Möglichkeiten der noch jungen Molekulargenetik überschätzt habe (DFG 1975: 6 f.). Die „gesellschaftspolitischen Probleme“, die sich im Zusammenhang mit der Mutagenitätsbekämpfung auf dem Weg politischer Regulierung herauskristallisierten, habe man hingegen unterschätzt (ebd.: 11). Die Kommission musste ihren regulatorischen Ansatz also auf realisierbare Schritte herunterschrauben, und nahm angesichts der gegebenen ökonomischen und methodischen Möglichkeiten des ZLM Abstand von der Prüfung im Routineverfahren. Die Tests des ZLM wurden auf eine „begrenzte Anzahl von besonders wichtigen Substanzen“ eingegrenzt; es sollten nunmehr nur noch Methoden der Mutagenitätsprüfung für den Routineeinsatz entwickelt werden, aber nicht selbst eingesetzt werden. Die Ergebnisse der Arbeit sollten darüber hinaus in Kursen und Tagungen einer breiteren Fachöffentlichkeit zugänglich gemacht werden (DFG 1977b: 68). Parallel dazu machte sich die Kommission auf die Suche nach „möglichen praktischen Ansatzpunkten“ für den Umgang mit der „Problematik aller Folgen eventuell nicht verhinderter Mutationen“ (DFG 1972b: 147).

\section{Die Pränataldiagnostik als neue Form der Vorsorge ab 1970}

Im ersten Teil dieses Beitrags haben wir untersucht, wie die Frage der Sicherung menschlicher Lebensgrundlagen in einer technisierten Umwelt im Laufe der 1950er und 1960er Jahre zum dringlichen Thema medizinischer und biologischer Forschungsinitiativen wurde, wobei Senatskommissionen als Grundlage politischer Regulierungsbestrebungen eingerichtet wurden. Wir haben gezeigt, dass auch Karl Knörr und Henriette Knörr-Gärtner ihre Arbeiten in der Strahlenforschung und der Teratologie in diesem Kontext verorteten, bevor sie zu Pionieren der westdeutschen Pränataldiagnostik 
wurden. Im zweiten Teil des Beitrags zeigen wir nun, wie diese ökologische Perspektive in der Frühphase der Planungen zur Förderung der Amniozentese aufgegriffen wurde.

Weltweit arbeiteten 1970 bereits 121 Laboratorien auf dem Gebiet der Pränataldiagnostik (vgl. Murken 1972b: 11). In der Bundesrepublik waren die Knörrs ab Ende der 1960er Jahre eine von insgesamt neun Forschungsgruppen, die bereits mit Technik der Amniozentese und den anschließenden zytogenetischen Untersuchungen experimentierten (vgl. Murken \& Stengel-Rutkowski 1973), wobei die Entnahme von Fruchtwasser noch stark risikobehaftet war (Schroeder-Kurth 1989: 21). Entsprechend führte Karl Knörr die ersten Versuche noch an Frauen durch, die kurz vor einem ohnehin geplanten Schwangerschaftsabbruch standen (Nippert 1991: 52). Zur Verbesserung der Verfahren wurde daher in DFG-Einzelanträgen sowie unter anderem im Rahmen des DFG-Sonderforschungsbereichs „Klinische Genetik“ und des DFG-Schwerpunktprogramms „Biochemische Grundlagen der Populationsgenetik des Menschen" mehrfach um Förderung angesucht (vgl. Thomaschke 2014: 127; zum SP „Biochemische Grundlagen“ vgl. Cottebrune 2008: 235-240). Den Anstoß für eine größer angelegte und zentral koordinierte Förderung der neuen Technologie gab aber letztlich die Kommission für Mutagenitätsfragen. In diesem Abschnitt werden wir zeigen, wie die Kommission mit dem Verfahren ihre Methoden zu erweitern suchte und damit auf die wahrgenommene Lücke in bisherigen, staatlich gesteuerten technokratischen Regulierungsbestrebungen reagierte.

Die Kommission war im selben Jahr nach den oben beschriebenen inhaltlichen und methodischen Debatten neu besetzt worden; Henriette Knörr-Gärtner gehörte ihr nun an (vgl. Schwerin 2010a). Anhand der Akten der DFG-Kommission lässt sich allerdings nicht belegen, dass sie merkliche Impulse zur Förderung der Pränataldiagnostik gegeben hätte. Vielmehr setzte sich vor allem der neue Vorsitzende der Kommission, der umtriebige und renommierte Molekularbiologe, Genetiker und Evolutionstheoretiker Carsten Bresch erfolgreich für die Förderung des Verfahrens ein. Bresch initiierte zwei internationale Symposien zur Amniozentese und erwirkte Ende 1972 den Beschluss zur Förderung des SP „Pränatale Diagnostik genetisch bedingter Defekte“, in dessen Rahmen von 1973 bis 1979 die Etablierung der genetischen Pränataldiagnostik in Westdeutschland erfolgte. Diese „Erweiterung“ der Mutagenitätsforschung zur Prävention einer befürchteten Verschlechterung des Erbguts ging mit einer Methodenverschiebung in der Vorsorge einher, was wir in den folgenden Abschnitten chronologisch darstellen werden. 
Carsten Bresch und die Amniozentese als „erweitertes Aufgabengebiet” der Mutagenitätsprüfung

In der Kommission für Mutagenitätsfragen begann man laut über Alternativen in der Prävention von Mutagenität nachzudenken, als Carsten Bresch im Herbst 1971 den Vorsitz der Kommission übernahm. Bresch hatte in den 1950er Jahren ein wichtiges molekulargenetisch orientiertes Forschungsinstitut in Köln aufgebaut und arbeitete zeitweise in den USA, wo er die Biology Division des Southwest Center for Advanced Studies im texanischen Dallas leitete (zum Kölner Institut vgl. Wenkel \& Deichmann 2007). Wie viele seiner Kollegen speziell in den USA, interessierte er sich für den Prozess der Evolution im Licht großer Zeitfragen: den Erhalt von Populationen, Lebensräumen und Ressourcen in einer Zeit zunehmenden Umweltbewusstseins und des Ost-West-Konflikts im Kalten Krieg (Bresch 1977; Krige 2002; Aronova et al. 2010; Little 2012). Für den bundesrepublikanischen Kontext stand Bresch für ein evolutionsbiologisch geprägtes Konzept von „Umwelt“ als „Außenwelt“, die durch den Menschen verändert werden könne und im Gegenzug auf das Leben und die Entwicklung der Organismen wirkte (Bresch 1977: 230, 233). Wenngleich direkte Bezüge nicht nachzuweisen sind, liegen gewisse Parallelen zu Richard Lewontins Konzept des constructed environment nahe. Zudem stand Bresch in der bundesdeutschen Öffentlichkeit für eine (auch popularisierende) Auseinandersetzung mit der vor allem in US-Großforschungsprojekten erprobten Idee, mittels kleinteiliger zytogenetischer, biotechnischer, biochemischer und serologischer Technologien Daten zu produzieren und zu vernetzen, um große Fragen nach der „biologischen Zukunft“, der Sicherung von Gesundheit und Wohlstand des Menschen für die Zukunft zu beantworten. ${ }^{4}$

Als erfahrener Wissenschaftsmanager und Experte auf dem Gebiet der Umweltforschung war Bresch 1971 von der Bundesregierung als Gutachter herangezogen worden, um die zukünftige „Organisation der wissenschaftlichen Beratung der Bundesregierung in Umweltfragen“ zu entwerfen (vgl. Materialienband zum Umweltprogramm 1971: 567-585). In dem Gutachten hatte er die "Schädigung menschlichen Erbgutes durch bestimmte chemische Substanzen (Mutagene)" als eine der dringlichsten Umweltgefahren der Gegenwart eingestuft, deren Untersuchung daher besonders gefördert werden müsse (ebd.: 568). Zugleich war er sich bewusst, dass die zuvor geschilderten Unsicherheiten im Bereich der Mutagenitätsprüfung ein Hindernis für die angestrebte Entwicklung neuer Instrumente der Regulierung darstellten. Er selbst war zwar von „natürlichen Schutzmechanismen gegen Mutationen“ überzeugt und verstand darunter „Reparatur-Prozesse an der genetischen Struktur“ oder "Selektion durch Spontanabort“ (DFG 1975: 7). ${ }^{5}$ Zugleich betonte er aber, dass zu wenig darüber bekannt sei, wie RepairMechanismen in vitro sich zu jenen in vivo verhielten, wie aussagekräftig 
diesbezüglich Tierversuche einzuschätzen seien und wie etwa sozioökonomische Faktoren sogenannter Subpopulationen (z.B. Berufsgruppen) auf die „Mutagenitätsstärke“ wirkten (ebd.: 6-10). In Anbetracht all der Unwägbarkeiten, die die bisherige Arbeit der Kommission geprägt hatten, legte Bresch im November 1971 dem zuständigen DFG-Referenten Pläne vor, die Mutagenitätsforschung um ein „Aufgabengebiet“ zu erweitern (DFG 1972b: 173):

Die Veränderung der menschlichen Umwelt unter dem Einfluß einer fortschreitenden technischen Entwicklung birgt vielerlei GefahrenMomente, die seit einiger Zeit in Wissenschaft und Öffentlichkeit reges Interesse finden. Relativ wenig beachtet - weil zu wenig erforscht - bleiben dabei Aspekte, die die Gefährdung des menschlichen Erbguts betreffen. Im Gegensatz zu den meisten anderen Umwelt-Problemen ist aber gerade diese Gefahr dadurch ausgezeichnet, daß heute begangene „Sünden“ erst im Laufe vieler zukünftiger Generationen wiedergutgemacht werden könnten. Nachlässigkeit oder Sorglosigkeit auf diesem Gebiet mag bezahlt werden müssen mit Tausenden und abertausenden von bedauernswerten menschlichen Kreaturen, deren körperliche oder geistige Funktionen durch defekte Erbanlagen gestört sind. Der Schutz des menschlichen Erbguts vor der Gefahr einer laufenden Anreicherung von genetischen Defekten infolge von Umwelt-Mutagenität ist daher jede wissenschaftliche Anstrengung wert (Bresch/Neumann an Klofat, 25.11.1971).

Bresch skizzierte als Bedrohungslage also eine Zunahme von Menschen mit Behinderungen (hier in diskriminierender Rhetorik als „bedauernswerte[n] menschliche[n] Kreaturen" bezeichnet) durch eine stetige Verschlechterung des Erbguts durch Umweltnoxen („laufende Anreicherung von genetischen Defekten") in einer zunehmend technisierten Welt. Das hier aufgegriffene Konzept genetischer „Degeneration“ hat bekanntlich eine längere Geschichte (vgl. Weingart et al. 1988: 27-91; Etzemüller 2007). Nach dem Zweiten Weltkrieg erfuhr es in den Sorgen um das Überleben der Menschheit in einer technisierten Umwelt eine Wiederbelebung und bildete hier Teil des beschriebenen Motivationsschemas, mit dem zahlreiche Forschungsprojekte auf dem Gebiet der Humangenetik und der Umweltforschung begründet worden waren. Bresch machte nun in seinem Schreiben gegenüber der DFG den Vorschlag, einer solchen Verschlechterung des Erbguts mit einem „Warnsystem und Bestimmung der Normalsituation", mit "Schutz und Abwehrmaßnahmen“ sowie „Gegenmaßnahmen“ zu begegnen. ${ }^{6}$ Mit dem Vorschlag der „Schaffung eines FrühWarnsystems für den allerdings extrem unwahrscheinlichen Fall des Eintretens einer genetischen Katastrophe (rapider Anstieg der Mutationsra- 
ten aufgrund undurchdachter und unvorhersehbarer Folgen technischer Neuerungen)“ (ebd.) verwies Bresch auf ein atomares Vernichtungsszenario im Zusammenhang mit dem Ost-West-Konflikt. Mit seinem zweiten Vorschlag zielte er hingegen auf den zivilen Alltag: die „Entwicklung von Verfahren zur laufenden Reinigung des Erbguts der menschlichen Population von schädlichen Defekten, um im Ernstfalle wirksame Maßnahmen zur Verfügung zu haben, einer steigenden Häufigkeit von Mißgeburten entgegenwirken zu können“ (ebd.). Hierfür biete sich, schrieb Bresch konkret, „die vor kurzem in den USA entwickelte Technik der Amniocentese an“ (ebd.).

Bereits einige Jahre zuvor hatte Bresch in seinem Lehrbuch die genetische Forschung als ein Mittel zur Sicherung der „biologischen Zukunft“ in Position gebracht (Bresch 1964: 66, 312). Etwa zur gleichen Zeit wurden im Deutschen Ärzteblatt erstmals Forschungen zur pränatalen genetischen Diagnostik erwähnt und über Spekulationen internationaler Experten hinsichtlich möglicher Einsatzgebiete der Technik berichtet: zur Bestimmung sowohl umweltbedingter Anomalien (die von Strahlenbelastung, Virus-Infektionen oder Medikamenteneinnahme in der Frühschwangerschaft herrührten) als auch auf genetische Erkrankungen in der Familie zurückzuführender Anomalien (Unbekannt [„yx“] 1966). In der Zwischenzeit waren diese damals noch vagen Vorstellungen unter anderem durch die Arbeiten des Ehepaars Knörr in den klinisch-angewandten Bereich gerückt. Die Entnahme und Analyse embryonaler Zellen bei fortbestehender Schwangerschaft bargen nun ein enormes Versprechen: den Zugang zum heranwachsenden Kind im Mutterleib und somit präzise Aussagen über chromosomale Anomalien in der folgenden Generation. Um dies in Anspruch nehmen zu können, um die „entsprechenden medizinisch-naturwissenschaftlichen Forschungen voranzutreiben, die für eine einwandfreie Diagnose und gefahrlose Eingriffe erforderlich sind“, schloss Bresch sein Schreiben mit der Bitte um Förderung einer „Pilot-Studie zur Computer-Analyse menschlicher Chromosomen".7 Gemeint war damit die computerunterstützte Auswertung von Karyogrammen, die im selben Jahr in den USA erprobt worden war und rasch auch in der deutschen Wissenschaft und Öffentlichkeit als zukunftsträchtig galt, zumal sie einen Schwangerschaftsabbruch nach erfolgter Diagnostik zu einem deutlich früheren Zeitpunkt erlaubte (vgl. ebd.).

Modifikation der „Kontrolle von Umwelt-Mutagenität”: die Amniozentese als "Schutz und Abwehrmaßnahme”

Die DFG holte drei Gutachten zu Breschs Antrag und seinem Konzept eines zweistufigen Umweltmonitorings („Warnsystem“ auf der einen, „Schutz und Abwehrmaßnahmen“ sowie "Gegenmaßnahmen" mit Hilfe der Amnio- 
zentese auf der anderen Seite) ein. Die Gutachten des Biochemikers Hansjürgen Staudinger und des ebenfalls biochemisch arbeitenden Humangenetikers Heinz-Werner Goedde fielen ohne nennenswerte Einschränkungen positiv aus. Der dritte Gutachter hingegen zeigte sich weniger überzeugt. Es handelte sich um den renommierten Heidelberger Humangenetiker Friedrich Vogel (1925-2006). ${ }^{8}$ Vogel war eine Schlüsselfigur der bundesdeutschen Humangenetik, er galt als Vertreter eines klinisch-laborwissenschaftlichen Zugangs, der sich an Erkenntnissen seiner US-amerikanischen Kollegen orientierte (Cottebrune 2012: 58 f.) und mit seinem Lehrbuch für genetische Familienberatung relativ früh das Ziel einer nichtdirektiven Beratung von Paaren und Familien zum Verhindern einer „Ausbreitung krankhafter Erbanlagen in zukünftigen Generationen“ propagierte (Vogel \& Fuhrmann 1968). Er leitete ab 1962 das Heidelberger Institut für Humangenetik und baute es in den folgenden Jahren zu einem Zentrum der Mutagenitätsforschung in Westdeutschland aus - zur weiteren Umgestaltung des ZLM sollte er bald darauf folglich auch als Experte hinzugezogen werden. ${ }^{9}$ Entsprechend war er in seiner Gutachterrolle mit den regulationspolitischen Anforderungen an die Kommission für Mutagenitätsfragen vertraut. Die Vorstellungen von einer angemessenen Herangehensweise an die Mutagenitätsprüfung gingen jedoch weit auseinander. Vogel und sein Mitarbeiter Röhrborn vertraten einen säugetiergenetischen Ansatz, der im zunächst molekulargenetisch konzipierten ZLM nur zögerlich integriert wurde (vgl. Schwerin 2012b). So erklärt sich vielleicht auch, weshalb Vogel den Vorstoß des Molekulargenetikers Bresch kritisch begutachtete.

Anders als Goedde und Staudinger zeigte sich Vogel also von Carsten Breschs Überlegungen nur teilweise überzeugt. Die Vorstellung der Machbarkeit eines umweltmutagenetischen Monitorings oder gar eines entsprechenden „Frühwarnsystems“ kritisierte er mit Verweis auf die ernüchternden Erfahrungen aus dem teratologischen SP „Schwangerschaftsverlauf und Kindesentwicklung“, in dessen Leitung er als stellvertretender Vorsitzender der Kommission für teratologische Fragen involviert war:

Was die Vorschläge Nr. 1. und 2. (Umweltmutagenität; Frühwarnsystem) betrifft, so würde ich den Vorschlag machen, hier die ganze Angelegenheit nocheinmal zu überdenken. Insbesondere der Vorschlag Nr. 2 (Frühwarnsystem) scheint mir doch von einem außergewöhnlichen Ausmaß von Naivität auf statistischem Gebiet zu zeugen. Vielleicht sollte man hier als ersten Schritt einmal eine Sitzung der Mutagenitätskommission einberufen, auf der ich selbst über diese statistischen Aspekte vortragen könnte (Vogel an Klofat, 19.01.1972).

Parallel zu der Begutachtung diskutierte die Mutagenitätskommission Breschs Entwurf in einer Sitzung Anfang Dezember 1971. Die im ZLM 
anwesenden Wissenschaftler waren vom Kommissionsvorstand informiert worden, dass die Planung eines Amniozentese-Programms „zum allgemeinen Aufgabenbereich der Kommission für Mutagenitätsfragen gehöre, auch wenn es nicht direkt etwas mit Mutagenität zu tun habe. Er [der Vorstand] betrachte es als Aufgabe der Kommission, alle Maßnahmen in die Wege zu leiten, die zum allgemeinen Schutz der Bevölkerung vor der Gefahr einer verbreiteten induzierten Mutagenese notwendig seien". ${ }^{10}$ Details wolle man nach einer „Amniozentese-Tagung“ bei Ulm im März 1972 mit Fachleuten anderer Disziplinen diskutieren. Die Nachfragen bezogen sich zum einen auf internationale Entwicklungen, etwa ob die Emergency Medical Services (EMS) oder die World Health Organisation (WHO) bereits „auf dem Gebiet aktiv seien“ (ebd.). Zum anderen ergänzten die Forscher, dass sich Überlegungen zu "Schutz- und Abwehrmaßnahmen“ gegen Umweltnoxen neben Strahlen mit Viren und Impfstoffen befassen müssten (ebd.). Kritischer äußerten sich die Anwesenden hingegen zum geplanten „Monitoring System“. Man vermutete „Schwierigkeiten der Erfassung" und verwies dabei, wie schon Vogel in seinem Gutachten, speziell auf die Erfahrung im SP „Schwangerschaftsverlauf“.

Um die Modifikation des Umweltmonitoringkonzepts in dieser Phase $\mathrm{zu}$ verstehen, muss berücksichtigt werden, dass Monitoringverfahren zu dieser Zeit auch in der Mutagenitätsforschung keineswegs unbekannt waren und in den folgenden Jahren als klassisches Top-down-Modell der Regulierung weiter verfolgt wurden (DFG 1977c). Die bundesrepublikanische Öffentlichkeit stand jedoch Vorstellungen der Vorsorge durch Überwachung, wie sie in der DDR umgesetzt und vielfach als kollektivistisch aufgefasst wurden, grundsätzlich skeptisch gegenüber (Madarász 2010). Ein vom Atomministerium finanziertes Monitoring der Mutationsrate der Gesamtbevölkerung war gescheitert (Kröner 1997), die 1970 implementierte postnatale Untersuchung auf Phenylketonurie stellte im Bereich der klinischen Genetik die Ausnahme eines weitgehend unhinterfragten und akzeptierten Screenings dar (Osten 2010; vgl. auch Löwy 2014b). Entsprechend schlug auch Vogel einen anderen Weg vor, nämlich die Fokussierung auf die Amniozentese, und fügte als Argument dafür hinzu:

Es ist auch mir bekannt, daß sich die Rechtslage bezüglich der genetischen Indikation zur Schwangerschaftsunterbrechung in der Bundesrepublik in Zukunft ändern dürfte. Aus diesem Grund und wegen der tatsächlich bestehenden Möglichkeit erscheint es auch mir dringend, dieses Arbeitsgebiet zu fördern. Der von Herrn Bresch vorgeschlagene Weg zur Förderung erscheint mir sehr geeignet, dieses Thema voranzutreiben (Vogel an Klofat, 19.01.1972). 
Wohl als Reaktion auf die ZLM-Sitzung rückte Bresch in den darauffolgenden Schreiben an die DFG stattdessen die pränatale Diagnose in den Mittelpunkt seiner Bestrebungen: „Die Erkennung einer Gefahr ist zwar eine notwendige aber noch keine hinreichende Bedingung ihrer Abwendung. Aus diesen Gründen erscheint die Förderung der Techniken zur pränatalen Diagnose von genetischen Defekten eine vordringliche Aufgabenstellung", argumentierte er, und beantragte die Förderung von zwei internationalen Arbeitstagungen auf dem Gebiet der Amniozentese. ${ }^{11}$ Diese Pläne fanden bei der DFG Gehör, die Arbeitsgespräche zur Vorbereitung eines entsprechenden Schwerpunktprogramms wurden bewilligt: „Die Öffentlichkeit wird an der Verfolgung dieser Projekte sicherlich großes Interesse zeigen; nicht nur, weil die Programme dieser Art zur Zeit ,en vogue', sondern weil die Sorgen der Genetiker berechtigt sind“, lesen wir in der internen Korrespondenz der Fachreferenten. ${ }^{12}$ Parallel zum Verblassen von Vorstellungen staatlich gesteuerter, technokratischer Gefahrenregulierung rückte in diesen Wochen also erstmals das Individuum als Interventionsobjekt in die Vorstellungen von genetischer Vorsorge ein.

Arbeitsgespräche auf Schloss Reisensburg und in Freiburg: Orientierung an sozial- und gesundheitspolitischen Entwicklungen

Das erste Arbeitsgespräch zur Präzisierung von Breschs Antrag auf prioritäre Förderung der pränatalen Diagnostik fand bald darauf vom 20. bis 22. März 1972 auf Schloss Reisensburg bei Günzburg an der Donau statt und versammelte Vertreter der inzwischen eingerichteten „Unterkommission Pränatale Diagnose genetischer Defekte der Senatskommission für Mutagenitätsfragen“ sowie der DFG-,,Prüfungsgruppe Pränataldiagnostik“. Mit Bresch, Winfried Krone, Rudolf Hausmann und Werner Schmid waren Lehrstuhlinhaber aus den Bereichen Humangenetik, Molekulargenetik und Gynäkologie anwesend. Das Thema, die „Pränatale Diagnostik genetischer Defekte“, war erneut in den breiteren Rahmen der „Kontrolle der Umwelt-Mutagenität" und der "Einrichtung eines Frühwarn-Systems“ gestellt worden. ${ }^{13}$ Man besprach zwar „eine Reihe von möglichen praktischen Ansatzpunkten (Routineuntersuchungen von Neugeborenen auf Homozygotie in wenigen rezessiven Erbleiden; Blutmutationen; Monitoringsystem auf internationaler Ebene; Frühsicherung von Gameten; Entwicklung eines Amniocentese-Programmes u.a.)" (DFG 1972b: 146 f.), doch wurden die Debatten zunehmend durch Fragen der Finanzierung geprägt. So wurden etwa den Kosten für ein „Musterinstitut" zur Pränataldiagnostik (inklusive des erforderlichen Personals sowie aller Geräte und Räumlichkeiten) Tabellen mit der Anzahl der in der BRD und in West-Berlin Lebendgeborenen sowie dem „rechnerisch ermittelten Anteil mongoloider Kinder in Abhängigkeit vom Alter der Mütter" gegenübergestellt. ${ }^{14}$ Damit zusam- 
menhängend wurde der Verlauf der Diskussionen von einem sich ankündigenden sozialpolitischen Wandel beeinflusst: die bevorstehende Reform der gesetzlichen Reglung zum Schwangerschaftsabbruch (die letztlich 1976 erfolgte) sowie, damit verbunden, die Aufnahme der Pränataldiagnostik in den Leistungskatalog der Krankenkassen als Perspektive zur längerfristigen Etablierung und Finanzierung des Verfahrens.

Ein bereits im Gutachten Friedrich Vogels angesprochener Umstand, der zu diesem Zeitpunkt die Einführung der Amniozentese hemmte, war die rechtlich unklare Situation, die sich ergab, wenn Mediziner nach gestellter Diagnose ihren Patientinnen tatsächliche Maßnahmen empfehlen wollten. Schließlich gab es für den überwiegenden Teil der durch die Chromosomenanalysen und biochemischen Tests diagnostizierbaren Schäden zum damaligen Zeitpunkt (wie auch heute noch) keine Therapie. Die Debatten zur Liberalisierung der Abtreibung hatten international nach Bekanntwerden der Thalidomid-Schädigungen, in der Bundesrepublik verhältnismäßig spät, nach 1968, an Intensität gewonnen und erhielten durch Argumente führender Mediziner in Folge wichtige Impulse (vgl. für die BRD: Nemec \& Moser 2017; international: Löwy 2014a: 158 f.). Der junge Kinderarzt Jan Murken hatte beispielsweise die Proceedings einer von ihm schon 1970 in München veranstalteten Tagung in einem dünnen Bändchen herausgegeben (vgl. Murken 1972a) und in dieser Publikation Anwendungszusammenhänge in der genetischen Beratung sowie ethische und rechtliche Aspekte der Schwangerschaftsunterbrechung als hochaktuelle ethische und juristische Fragen benannt (ebd.). Er selbst hatte in der Einleitung programmatisch hervorgehoben, dass die erwartete Gesetzesänderung den selektiven Schwangerschaftsabbruch auf Grund „genetischer Indikation“ vorsehe (Murken 1972b: 12 f.). Der ebenfalls an der Tagung beteiligte Karl Knörr hatte zwar über einen Einsatz der Amniozentese im kurativen Bereich spekuliert (Knörr 1971: 620), ging in seinem Beitrag zugleich so weit, sie für die gynäkologische Praxis als „nur dann sinnvoll zu bezeichnen, wenn die Möglichkeit zur pränatalen Therapie oder zur Durchführung der Interruption graviditas besteht“ (Knörr 1972: 66). Zudem zeigte er sich überzeugt, dass die Pränataldiagnostik dem Staat „die Entscheidung zur Erweiterung der Indikation zur Interruptio graviditas eher erleichtern“ dürfte (ebd.: 67). Bresch hatte entsprechend bereits in seinem ersten Schreiben an die DFG auf die laufenden Debatten zur Reform des betreffenden $\$ 218$ StGB verwiesen und vermerkt, er sei überzeugt, dass „mit Sicherheit die genetische Indikation einen Abort zulassen" und folglich die Amniozentese bei „Schwangeren mit hohem Risiko für Defektgeburten [...] zu einer Routine-Procedur wird“. ${ }^{15}$ Auf Schloss Reisensburg konkretisierte Bresch diese Zielsetzung: „Die Arbeitstagung der Deutschen Forschungsgemeinschaft zu Planungsfragen im Bereich der pränatalen Diagnose soll helfen, 
rechtzeitig die erforderlichen Maßnahmen einzuleiten, die durch die zu erwartende Änderung des $\$ 218$ auf uns zukommen."

In forschungsstrategischen Überlegungen kamen die Anwesenden zudem zu dem Schluss, dass es bezüglich der Finanzierung der Etablierung der Pränataldiagnostik am zielführendsten sei, sie bei den Krankenkassen als „ein Aspekt der Präventivmedizin“, beim Bundesministerium „als flan-

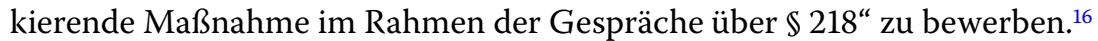
Diese Entscheidung, die genetische Pränataldiagnostik als sogenannte Vorsorgeleistung zur Prävention von Behinderung oder Beeinträchtigung zu bewerben, hatte den Hintergrund in einer spezifischen Struktur des westdeutschen Gesundheitssystems, das, wie die Historikerin Ulrike Lindner gezeigt hat, nahezu gänzlich auf individuell abrechenbare Leistungen fokussierte, und damit vor allem auf die Therapie (hierzu vgl. Lindner 2007). Zudem waren wenige Monate vor der Tagung gewisse Vorsorgeleistungen im Zuständigkeitsbereich der gesetzlichen Krankenversicherung verankert worden (Stockhausen 1971). Vor diesem Hintergrund schien die Strategie vielversprechend, ein zu diesem Zeitpunkt noch mehrheitlich umwelthygienisch gerahmtes Ziel, die Prävention von genetischen Anomalien, durch die Pränataldiagnostik als individualisiertes Verfahren in den Leistungskatalog der gesetzlichen Krankenkassen und damit in den Alltag von Kliniken und niedergelassenen Ärztinnen und Ärzten zu implementieren. Diese Strategie sollte letztlich Erfolg zeigen: Schon 1975 wurde die Amniozentese als Präventivleistung in den Katalog der Krankenkassen aufgenommen (Mutterschafts-Richtlinien 1974). Gemäß dem nach verwickelten Debatten letztlich beschlossenen, Gesetz zur Neuregelung des Schwangerschaftsabbruchs von 1976 wurde dieser straffrei gestellt, „wenn nach ärztlicher Erkenntnis [...] dringende Gründe für die Annahme sprechen, daß das Kind infolge einer Erbanlage oder schädlicher Einflüsse vor der Geburt an einer nicht behebbaren Schädigung seines Gesundheitszustandes leiden würde“ (Fünfzehntes Strafrechtsänderungsgesetz 1976; zu den Debatten um $\$ 218$ vgl. Behren 2004). Die Frist, innerhalb derer die Indikation zugelassen war, betrug 22 Wochen - genau die Zeitspanne, die zur verlässlichen Durchführung einer Amniozentese mit pränataler Diagnose benötigt wurde.

Ziel des zweiten Treffens am Institut für Biologie in Freiburg, das am 14. Mai 1972 stattfand, war die Evaluation der bundesrepublikanischen Debatten unter ausländischen Kolleginnen und Kollegen, darunter Daniel Bergsma (White Plains, N.Y.), Victor A. McKusick (Baltimore, MD), Margareta Mikkelsen (Glostrup) und Jarvis Edwin Seegmiller (San Diego, La Jolla). Das Gespräch fand im Anschluss an die Jahrestagung der Gesellschaft für Humangenetik zur „Frühdiagnose genetisch bedingter Anomalien beim Menschen" statt, wobei die dort gehaltenen Referate der deutschen Forscher bereits die inhaltlichen Eckpunkte des im Anschluss angesetz- 
ten internationalen Hearings markierten und verdeutlichten, dass dieses der unmittelbaren Vorbereitung des Forschungsantrags dienen würde: Karl Knörr referierte über „Techniques of Amniocentesis“, Karl-Heinz Degenhardt über den Einsatz der Amniozentese in der genetischen Beratung; der Genetiker Widukind Lenz leitete ein öffentliches Podiumsgespräch zu juristischen, sozialen und ethischen Aspekten der Pränataldiagnostik (vgl. auch DFG 1973: 173; Nippert 1991: 56 f.). ${ }^{17}$ Carsten Bresch hatte vorab einen Fragebogen konzipiert und die Teilnehmer gebeten, über Erfahrungen mit dem Aufbau von Diagnostikeinrichtungen, mit Formen der nationalen und übernationalen Koordination des Austauschs von Forschergruppen, aber auch über Detailfragen zum Austausch ihrer Materialien (etwa dem Versand von Zellkulturen mit dem Flugzeug) zu berichten. ${ }^{18}$ Doch bestand zudem, nachdem man sich auf Schloss Reisensburg geeinigt hatte, die Amniozentese als Präventivleistung zu bewerben, ein großes Interesse $\mathrm{zu}$ erfahren, wie man die Nachfrage von Patientinnen steigern könne, welche „Popularisierungsstrategien“ und „Wege die Öffentlichkeit zu überzeugen“ eingeschlagen werden konnten. ${ }^{19}$ Die „attitude of the physicians is important, then the demand will increase sharply“, resümierte Werner Schmid die Ergebnisse der Debatte. „Our planning has to be connected with a genetic counselling dep.[artment]“, fügte Friedrich Vogel hinzu (ebd.), und machte die inzwischen vorrangige Orientierung des in der Mutagenitätsforschung gestarteten Programms an praktischen Fragen der Implementierung in der klinischen Genetik und der Gynäkologie deutlich.

Abschlussbesprechung im Frankfurter Hotel Intercontinental und der Beschluss des „Amniocentese-Programms”

In förderpolitischer Hinsicht entscheidend war schließlich eine Abschlussbesprechung im Frankfurter Hotel Intercontinental am 17. November 1972. Die DFG hatte eine „Besprechungsgruppe“ von Entscheidungsträgern geladen, ${ }^{20}$ um festzulegen, welche konkreten Fördermaßnahmen im „Problemkreis der vorgeburtlichen Diagnostik" bearbeitet werden und welche Arbeitsgruppen an diesem Vorhaben teilhaben sollten. ${ }^{21}$ Die am Ende des Treffens ins Auge gefassten drei Einsatzgebiete der Amniozentese waren nun, geprägt durch die Ergebnisse des internationalen Hearings, zulasten der umwelthygienischen Perspektive geändert worden und waren ausgerichtet auf: das Erkennen „spontaner Mutationsraten“ durch Umwelteinflüsse, „Ehen mit genetischen Problemen“ und das „hohe Alter der Schwangeren“. ${ }^{22}$ Allerdings formulierte Bresch, wie aus einer abschließenden $\mathrm{Zu}$ sammenfassung der Ergebnisse der Arbeitsgespräche hervorgeht, vor allem praxisbezogene Aspekte als „Motive der DFG-Senatskommission für $\mathrm{Mu}$ tagenitätsfragen, der DFG eine Förderung der Techniken der Pränatalen Diagnose von genetischen Defekten zu empfehlen“: 
Es sei zu erwarten, daß auch in der Bundesrepublik Deutschland in absehbarer Zeit eine Schwangerschaftsunterbrechung bei Vorliegen eines schwerwiegenden genetischen Defektes erlaubt sein werde. Dadurch werde es bei vielen Ehen mit genetischen Problemen möglich sein, zu gesunder Nachkommenschaft zu verhelfen. Das Verfahren der Amniocentese (Fruchtwasserpunktierung) sei wissenschaftlich noch problemgeladen und bedarf daher noch eingehender Untersuchungen (ebd., Hervorhebung im Original).

Knörr fügte hinzu, eine Gesetzesänderung im Sinn einer Frist zum straffreien Abbruch bis zur 14. Woche sei, wie das Beispiel der USA zeige, für die Diagnostik ausreichend und zugleich für die Forschung wichtig, denn es würde diese mit „Übungsmaterial“ versorgen (ebd.).

Ein weiteres Ergebnis des Treffens im Intercontinental war zudem die deutliche Herausbildung einer Forschungsgemeinschaft zur Pränataldiagnostik, eine „kleine Gruppe von Wissenschaftlern“ - Genetikern, Humangenetikern, Cytologen, Biochemikern, Gynäkologen, Pädiatern und Juristen (ebd.), wobei Bresch die Gynäkologie als zentrale Disziplin des Programms festlegte. Durch seine Unterstützung war Karl Knörr in dieser Sitzung bereits in eine zentrale Position gerückt und bot an, „ein entsprechendes Formular für alle Ärzte vorzubereiten, die Amniocentese durchführen werden“ (ebd.). Die jeweiligen Vorarbeiten der Teilnehmer, speziell aus dem Teratologie-Projekt, sollten in die konzeptuellen Überlegungen einfließen. KarlHeinz Degenhardt etwa brachte Erfahrungen aus der Schwangerschaftsverlauf-Studie ein, speziell in Fragen der Standardisierung von Techniken und Normwerten, des Ausbaus und der Umgestaltung von Laboratorien, der Schulung von Personal und Hilfskräften sowie der Koordination von Arbeitsbesuchen im Ausland (ebd.; zu diesem Transfer vgl. Murken \& Stengel-Rutkowski 1974). Das Teratologie-Projekt stand für die Anwesenden zugleich aber auch für die Probleme zentraler Erfassung von $\mathrm{Pa}-$ tienten- und Beobachtungsdaten. Wesentlich vielversprechender erschien es ihnen daher, wie der weitere Verlauf des Protokolls zeigt, klinisch-praktische Aspekte in den Vordergrund zu stellen, um die „wissenschaftlichen Voraussetzungen zu schaffen, damit diese Amniocentese-Untersuchungen gefahrlos und mit Sicherheit in der Aussage durchgeführt werden können“.

Der Beschluss zur „vorrangigen Förderung“ der Amniozentese fiel nur kurze Zeit später, im Dezember 1972, ${ }^{23}$ nachdem die Priorität des Schwerpunkts von weiteren Experten, darunter die sogenannte Planungsgruppe für die Biowissenschaften, bestätigt worden war (ebd.). Wohl auch um eine Brücke zur Grundlagenforschung, dem eigentlichen Fördergebiet der DFG, herzustellen, legte man fest: 
Im einzelnen werden bevorzugt Projekte gefördert, die folgenden Themenkreisen zugeordnet werden können: Techniken der Amniocentese; Technik der Zellkultur von Aminionzellen; Biochemisch-genetische Untersuchungen in Zellkulturen; Immunologische Beziehungen zwischen Mutter und Foet; Cytogenische Techniken; Längsschnittuntersuchungen des Kindes nach Amniocentese (DFG 1974: 78).

Spätere Quellen bestätigen jedoch, dass die erwartete Reform des $\$ 218$ und dessen Aufnahme in den Leistungskatalog der Krankenkassen die Entscheidung der DFG letztlich maßgeblich beeinflussten. Es wurde „im Hinblick auf die große wirtschaftliche Bedeutung dieses Programms [...] in Kauf genommen“, den Aufbau einer individualmedizinischen Routineleistung aus Fördertöpfen zu finanzieren, die eigentlich für die Grundlagenforschung bestimmt waren, und zudem zunächst jene Kosten zu übernehmen, die nicht von den Kassen erstattet würden, erläuterte Jan Murken rückblickend (Murken 1978: 1381; vgl. Nippert 1991: 57). ${ }^{24}$ Zudem war es Ziel durch die Förderung „rechtzeitig die erforderlichen Maßnahmen von Seiten der Wissenschaft einzuleiten, die durch die wahrscheinliche Änderung des $\$ 218$ StGB hinsichtlich der Unterbrechungsmöglichkeit einer Schwangerschaft aufgrund eines zu erwartenden genetischen Defektes des Kindes notwendig werden würden", heißt es im Abschlussbericht des Programms (zitiert nach Stengel-Rutkowski et al. 1992: 1).

Als Quintessenz lassen sich aus den Protokollen der Arbeitsgespräche zwischen Dezember 1971 und November 1972 ein Verblassen von Vorstellungen staatlich gesteuerter, technokratischer Gefahrenregulierung, wie sie am ZLM weiterhin praktiziert wurde, und eine Veränderung dieses regulatorischen Ansatzes herauslesen. Dieser Wandel zeigt sich am deutlichsten in dem Einrücken des Individuums als Interventionsobjekt in die Politik und Praxis der genetischen Vorsorge und durch die Hinwendung zu den Bedürfnissen der klinischen Praxis. Im Rahmen der Senatskommission für Mutagenitätsforschung, die noch dem Zeitgeist der Planbarkeit, der Steuerung durch Expertenwissen, entsprang, war vor dem Hintergrund eines sozialhistorischen Wandels im Zusammenhang mit der Reform des $\$ 218$ und einer gesundheitspolitischen Wende im Zuge der Aufnahme von Präventivleistungen in den Katalog der Krankenkassen eine bemerkenswerte Transformation erfolgt: eine Modifikation der „Kontrolle von UmweltMutagenität" durch die Fokussierung auf die Amniozentese als „Schutz und Abwehrmaßnahme" anstelle eines populationsgenetischen "Warnsystems" - oder, anders gesagt, auf eine klinisch-praktische Lösungen für ein vormals umwelthygienisch gerahmtes Problem. Die Entstehung der genetischen pränatalen Diagnostik aus der Mutagenitätsforschung hatte in inhaltlichen und methodischen Debatten, die die Arbeit der Mutagenitäts- 
Kommission sowohl in wissenschaftlicher als auch in regulationspolitischer Hinsicht prägten, ihren Ursprung und resultierte in einer Methodenverschiebung in der Vorsorge: einer Individualisierung von Vorsorge durch die Einführung eines diagnostischen Verfahrens, dessen Etablierung hierauf in Kliniken, genetischen Beratungsstellen und Privatpraxen im Rahmen des Schwerpunktprogramms „Pränatale Diagnostik“ begann (zur Umsetzung vgl. Murken \& Stengel-Rutkowski 1973-1982).

Der Aufbau des DFG-Schwerpunktprogramms: Orientierung an klinisch-praktischen Anwendungen und das Verblassen der umweltmutagenetischen Perspektive

In den folgenden Wochen erfolgte der strukturelle Aufbau des Pränataldiagnostik-Programms: Karl Knörr wurde als inhaltlicher Leiter benannt; Jan Murken als zentraler Koordinator am anerkannten Zentrum für Kinderheilkunde des Universitätsklinikums München. Carsten Bresch hingegen zog sich in die weitere Umgestaltung des ZLM zurück: „Ich glaube aber, daß durch Einrichtung dieses Schwerpunktprogramms doch die Dinge jetzt ins Rollen gekommen sind“, schrieb er an die DFG. ${ }^{25}$ Wenige Monate später, im Herbst 1973, nahm das „Amniocentese-Programm“ unter dem Namen „Pränatale Diagnostik genetisch bedingter Defekte“ seine Tätigkeit auf. Die DFG resümierte die Chronologie der Fokussierung auf die Amniozentese im Rahmen der Mutagenitätsforschung in ihrem Jahresbericht:

Trotz aller Anstrengungen im Bereich der Mutagenitätsprüfung ist eine Gefährdung der menschlichen Erbinformation durch Umweltchemikalien aus mehreren Gründen niemals völlig auszuschließen: 1. Naturgemäß können auch sorgfältige Prüfverfahren die mögliche Gefahr von Langzeitwirkungen nur ungenügend erfassen. 2., ebenso können synergistische Effekte verschiedener Substanzen wegen der riesigen Zahl von Kombinationsmöglichkeiten kaum jemals systematisch geprüft werden, 3. Schließlich bleibt die Übertragung der experimentellen Daten vom Kleinsäugetier auf den Menschen oder von menschlichen Zellkulturen auf den ganzen Organismus immer mit einer gewissen Unsicherheit behaftet. Neben der Anstrengung, mutagene Substanzen rechtzeitig zu erkennen und aus dem Verkehr zu ziehen, müssen Abwehrmaßnahmen getroffen werden, um eine Gefährdung des Menschen für den Fall auszuschließen, daß aus einem der angeführten Gründe eine gewissenhafte Mutagenitätstestung nicht ausreicht. Aus solchen Erwägungen heraus hat sich eine Untergruppe der Kommission intensiv mit Fragen der pränatalen Diagnose genetischer Schäden befaßt. In zwei Arbeitsgesprächen mit internationalen Teilnehmern wurden alle in diesem Zusammenhang wichtigen Fragen erörtert. Dem Senat 


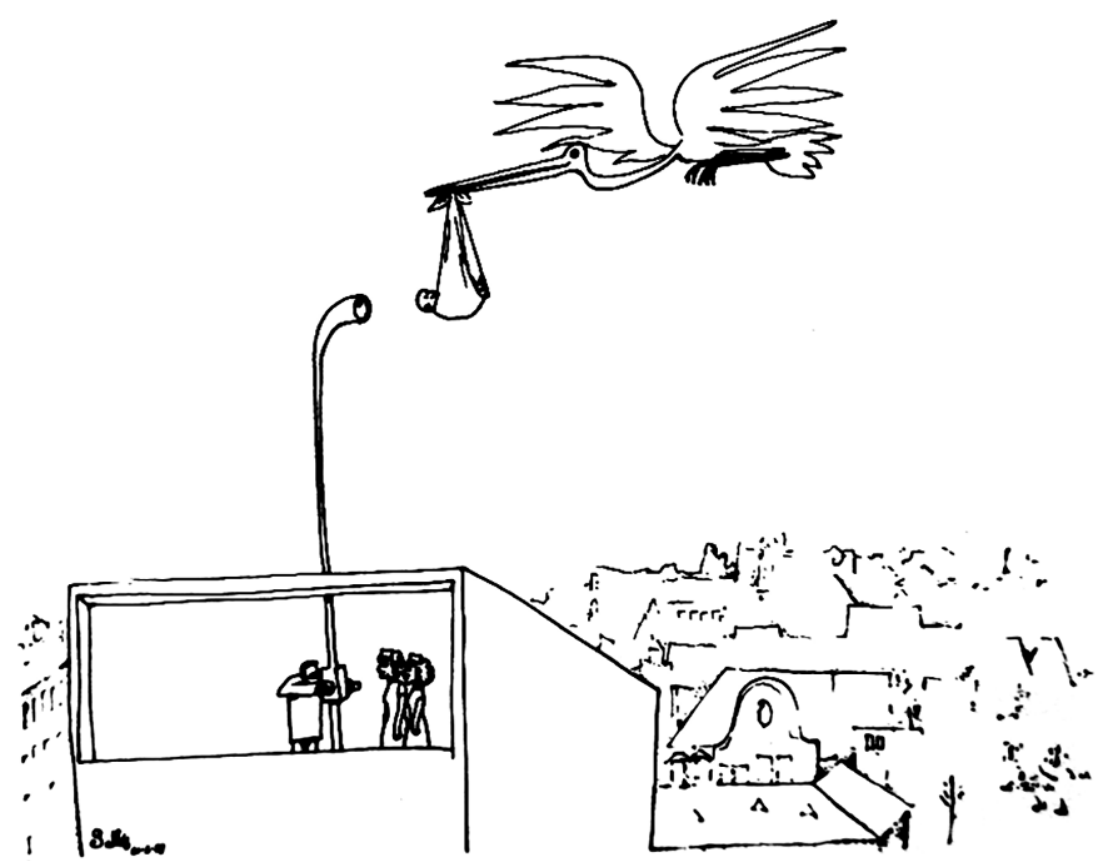

Abb. 2 Zeichnung im Einladungsfolder zur Tagung im April 1979 in München: ein Storch bringt den Wissenschaftler*innen das Kind zur Diagnostik, hier mit Hilfe eines Periskops. (Bundesarchiv in Koblenz, Ausschnitt aus dem Programmheft der Tagung; B 227/22509)

der DFG wurde aufgrund der Ergebnisse dieser beiden Besprechungen vorgeschlagen, einen Schwerpunkt zur Förderung dieses Gebietes einzurichten. Der neue Schwerpunkt „Pränatale Diagnose genetischer Defekte“ wird im Jahr 1973 anlaufen (DFG 1972b: 173).

Auf der im April 1979 in München stattfindenden 3. Europäischen Konferenz für pränatale Diagnose genetischer Defekte, mit der die Förderung des Programms durch die DFG endete, blickte man bereits auf das Erreichte zurück. Die pränatale Diagnose war von einer experimentellen Technik in eine Routinemethode überführt worden, was durch eine Zeichnung im Einladungsfolder auf humorvolle Weise thematisiert wurde: ein Storch legt bei der Auslieferung eines Kindes zur Diagnostik bei den Wissenschaftler*innen einen Zwischenstopp ein (Abb. 2). In diesem Sinne konstatierte der Bayerische Staatsminister Fritz Pirkl in der für ein internationales Publikum gehaltenen Eröffnungsansprache zur Konferenz: Dank der Pränataldiagnostik hätten die Humangenetik und die genetische Beratung "outgrown the research stage and [...] fully come up to the demands and requirements of practical medicine." Besonders erfreue 
ihn die "rapid acceptance of genetic counseling and its integration into the list of medical services of the social insurance scheme in the Federal Republic of Germany" (in: Murken et al. 1979: VII). Tatsächlich hatten im Rahmen des Schwerpunktprogramms, geeint durch ein gemeinsames Forschungsanliegen und die Förderung in Höhe von insgesamt ca. 9 Millionen DM, insgesamt etwa 200 Frauen- und Kinderärzte, Humangenetiker, Zytogenetiker und Biochemiker an über 30 Standorten auf dem gesamten Bundesgebiet Techniken und Methoden der Fruchtwasserentnahme, Zellkultur, Chromosomenanalyse und der biochemischen Analyse erlernt und verfeinert. Insgesamt 13.411 Amniozentesen mit anschließender Zellenzüchtung und Chromosomenanalyse bzw. biochemischer Analyse sowie genetischer Beratung fanden zwischen 1973 und 1979 jene finanzielle, strukturelle und moralische Unterstützung, die eine Etablierung als Routineverfahren bewirkte (Stengel-Rutkowski et al. 1992: 298-303) und damit den Grundstein für jene flächendeckende „Versorgung der Bevölkerung mit humangenetischen Leistungen“ legte, wie Ende der 1980er Jahre schließlich von Expert*innen konstatiert wurde (Schroeder-Kurth 1989; vgl. dazu Thomaschke 2014).

Mit der von Staatsminister Pirkl angesprochenen Anpassung an die Bedürfnisse der klinischen Praxis während der Laufzeit des DFG-Programms bestätigte sich die in den Arbeitsgesprächen bereits angestoßene Veränderung des regulatorischen Ansatzes: in der Praxis wurde die Pränataldiagnostik überwiegend zum Erkennen erblicher oder spontan auftretender genetischer Anomalien genutzt, wie die regelmäßigen Forschungsberichte des Programms zeigen. 1978 gestanden die Forscher ein, dass sie im Bereich der Prävention von Umweltgefahren nicht, wie ursprünglich erwartet, einzusetzen war:

Ohne Erfolg blieben die Versuche, Umwelteinflüsse wie Virusinfektion, Strahlenbelastung oder Medikamenteneinnahme in ihrer schädlichen Wirkung auf den Fetus pränatal zu diagnostizieren. Die Hoffnung, daß man vielleicht in Amniozentesekulturen induzierte Chromosomenbrüche finden könnte, die einen Rückschluß auf eine Schädigung des Fetus erlaubten, haben sich nicht erfüllt (Murken \& Albert 1978: 5).

Wurde in der Fachöffentlichkeit ein Einsatz der Amniozentese gegen exogene und erbliche Faktoren noch weiterhin diskutiert (Unbekannt [„Mst“] 1976: 724), wurden in der Praxis bereits 1979 über 80 Prozent der erfassten Diagnosen aufgrund der „Altersindikation“ gestellt (vgl. StengelRutkowski et al. 1992: 12). Das heißt, dass in Westdeutschland die Pränataldiagnostik - ausgehend von der Annahme, dass Spontanmutationen wie Trisomie 21 mit steigendem Alter der Eltern gehäufter auftraten - 
bereits um 1980 vorrangig eingesetzt wurde, um die Geburt von Kindern mit dieser Chromosomen-Anomalie zu verhindern.

Diese Diskrepanz hatte praktische Hintergründe. Ein Großteil der Frauen wandte sich an ihre Gynäkologen, weil sie sich wegen möglicher Erbkrankheiten und nicht wegen umweltbedingter Belastung Sorgen machten. Zwar war in den Erhebungsbögen des SP „Pränatale Diagnostik“ durchaus die Frage berücksichtigt worden, ob ein Elternteil eine „Exposition mit Strahlen, Viren, Chemikalien oder Mutagenen" erfahren hatte, doch unter den insgesamt 85 (von 13.411!) Fällen, in denen die Pränataldiagnostik wegen des Verdachts auf exogene „elterliche Mutagenbelastung“ durchgeführt wurde, fand sich kein einziger pathologischer Befund (Stengel-Rutkowski et al. 1992: 118). Es fehlte also die empirische Grundlage, um Aussagen über Umwelt-Mutagenität zu treffen. Diese Entwicklung auf Basis seiner Erfahrung in der gynäkologischen Praxis vorhersehend hatte Knörr bereits 1972 konstatiert, „der Schwerpunkt der pränatalen Diagnostik wird auf der Chromosomen-Analyse zum Nachweis oder Ausschluß von DownSyndrom liegen“ (Knörr 1972: 57). Auch Jan Murken hatte diesen Trend, dass Frauen nicht mehr überwiegend auf Grund des Verdachts umweltbedingter Belastung oder bekannter schwerer genetischer Erkrankungen in der Familie die Pränataldiagnostik nachfragten, an der Münchner Frauenklinik beobachtet:

Das Bewußtsein für genetische Fragestellungen in der Bevölkerung nimmt zu. Eine wichtige Rolle spielt dabei die Möglichkeit der Familienplanung: Wer die Zahl seiner Kinder nicht dem Zufall überläßt, wird sich auch Gedanken über die Gesundheit der gewünschten Kinder machen (Murken 1972a: 9).

In anderen Worten: Das Schwerpunktprogramm war also zum Schutz vor umweltbedingten Schäden am Erbgut von Wissenschaftlern unterschiedlicher Disziplinen beworben und umgesetzt worden, bevor die Amniozentese in einer späteren Phase ihrer klinisch-gynäkologischen Etablierung verstärkt im Zusammenhang mit der Reduktion der Geburt von Kindern mit spontan auftretenden Anomalien, wie Down-Syndrom, angeboten und in Anspruch genommen wurde.

\section{Fazit und Ausblick}

Die Amniozentese ist ein Verfahren, das Erforschung und Praxis der Reproduktion grundlegend veränderte. Als in den frühen 1970er Jahren die Entnahme und Diagnostik von fetalen Zellen aus dem Fruchtwasser auch 
bei fortbestehender Schwangerschaft möglich wurde, rückten genetische Tests von der Grundlagenforschung in den angewandten Bereich der genetischen Früherkennung. Die Professionalisierung und Institutionalisierung der Amniozentese erfolgte in Westdeutschland im Rahmen des DFGSchwerpunktprogramms „Pränatale Diagnose genetisch bedingter Defekte“ von 1973 bis 1979. In diesem Beitrag haben wir die Chronologie der ersten Vorarbeiten bis hin zur Beantragung dieses Programms nachgezeichnet und gezeigt, dass der Anstoß, ein „Amniocentese-Programm“ zu entwickeln, auf die DFG-Kommission für Mutagenitätsfragen zurückging und damit im Kontext von Forschungen zu Umwelteinflüssen auf das menschliche Erbgut entstand. Die bereits aus früheren Debatten bekannte Angst vor einer wachsenden Verschlechterung des Erbguts durch Umwelteinflüsse stand hierbei im Vordergrund. „Umwelt“ wurde in dieser Phase als ein durch „zunehmende Umweltbelastungen“ gekennzeichneter „menschlicher Lebensraum“ (DFG 1971a: 10) konzipiert, als durch den Menschen veränderte „Außenwelt“ (Bresch 1977), und folglich als wichtiger Gegenstand von Forschung und Politik. Als parallel zur Herausbildung eines globalen Umweltbewusstseins und eines in der Umweltgeschichte als „ökologische Wende“ (Radkau 2011) bezeichneten Bewusstseinswandels die Schaffung effektiver Initiativen und Institutionen der Vorsorge umso dringlicher wahrgenommen wurde, erschien die Amniozentese den beteiligten Forschern, speziell im Zusammenhang mit der sich ankündigenden Reform der rechtlichen Regelung des Schwangerschaftsabbruchs, als eine erfolgsversprechende „Schutzmaßnahme“. Erst in der anschließenden praktischen Umsetzung wurde der Fokus im Einsatz der Amniozentese auf die Reduktion erblicher, vor allem aber spontan auftretender Anomalien gelegt. Diese Verschiebung erfolgte durch Erfahrungen in der gynäkologischen klinischen Praxis und der Nachfrage von Frauen und Familien, die in den seltensten Fällen Umweltnoxen ausgesetzt waren. Die meisten wandten sich auf Grund des Wunsches, die Geburt eines Kindes mit Down Syndrom, einer spontan auftretenden genetischen Anomalie, zu verhindern, an die Gynäkologen. Diese Schritte einer Generalisierung der genetischen Diagnostik fanden in der Bundesrepublik parallel zu ähnlichen internationalen Entwicklungen statt: Seit den 1970er Jahren wurde die Pränataldiagnostik zu einem generalisierten Bestandteil der Schwangerschaftsvorsorge, wobei der Umstand, dass das Down Syndrom aus Sicht der öffentlichen Gesundheitsvorsorge mittlerweile ein zentrales Problem darstellte, eine wichtige Rolle spielte, was sowohl die medizinische Praxis als auch die Erfahrung von Schwangerschaft für Millionen Frauen und Familien weltweit maßgeblich veränderte (vgl. Löwy 2017).

Die hier dargestellte Fallstudie hat somit einige erste Befunde geliefert, an die sich weitere Forschungen anschließen müssten. So wäre zu unter- 
suchen, inwieweit die skizzierte Entwicklung in der Politik und Praxis der Vorsorge - im Sinn einer "Geburt der Pränataldiagnostik aus der Umweltforschung "26 - auch für andere Länder nachzuweisen wäre.

Ebenso wäre zu verfolgen, inwiefern die Etablierung einer klinisch-praktischen und individualmedizinischen Lösung für ein vormals umwelthygienisch gerahmtes Problem Teil einer größeren Entwicklung in der Medizingeschichte war, die in der Literatur als „präventivmedizinische Wende“ bezeichnet wird (vgl. Hähner-Rombach 2015; Schenk et al. 2013; Stöckel \& Walter 2002; Lengwiler \&Beck 2008). Für den Bereich der genetischen Beratung ist bereits nachgewiesen worden, dass eine Abkehr von Ätiologie und von Fragen nach krankheitsauslösenden Faktoren in der Umwelt um 1970 stattfand und dass diese Wende eng mit der Etablierung individualisierter Formen der Diagnostik, Vorsorge und Therapie zusammenhing. „Prävention“ diente in dieser Phase vor allem als wirkmächtiges Schlagwort zur Abgrenzung von früheren kollektivistischen Konzepten der Vorsorge, etwa der Umwelt-, Sozial- oder Rassenhygiene (Nemec \& Moser 2017; vgl. auch Harper \& Petermann 2017; Löwy 2014a). Zugleich hat die Forschung auf die rhetorische Inkonsistenz in Debatten führender Humangenetiker hingewiesen, in denen zwar eine Hinwendung zum Individuum, zur nichtdirektiven Beratung und die Unterstützung autonomer Entscheidungen gefordert wurde, zugleich aber diskriminierende Ansätze im Umgang mit genetischen Anomalien oder mit Behinderung, als wahrgenommene Gefahr und Kostenfaktor, gängig waren (Bösl 2009; Schenk 2013). Eine Wende von staatlich-kontrollierenden, paternalistischen, hierarchischen Zugängen zu autonomeren Vorstellungen des Individuums vollzog sich, wie unter anderem Anne Waldschmidt gezeigt hat, in der genetischen Beratung erst in den 1980er Jahren (Waldschmidt 1996; vgl. auch Maasen et al. 2011). Wie die Geschichte der Pränataldiagnostik in diesen größeren Wandel von wissenschaftlichen Debatten, aber auch von persönlichen Vorstellungen des Verhältnisses von Individuum und Population einzuordnen ist, lag außerhalb des Untersuchungsrahmens dieses Beitrags. Schließlich wäre eine interessante Frage, wie die Inanspruchnahme der Pränataldiagnostik ab den späten 1970er Jahren mit einem größeren gesellschaftlichen Wertewandel und der Entwicklung neoliberaler Vorsorgestrategien im Zusammenhang stand, wie sie Alexander von Schwerin für den Bereich der Strahlenforschung postuliert hat (Schwerin 2008: 189). Diese Fragen im Zusammenhang neuer Handlungsfelder der Gesundheitsforschung in der späten Bundesrepublik (vgl. zur Frühgeschichte Labisch \& Tennstedt 1991) oder der salutogenetischen Wende und erneuten Umweltdebatten der New Public Health zu Beginn der 1980er Jahre, etwa vor dem Hintergrund der Ottawa Charta der WHO zu diskutieren (Vgl. Leanza 2017: 220-258; Rosenbrock 1998), scheint uns eine vielversprechende Perspektive für weitere Arbeiten. 
Hinweis des Verlags Der Verlag bleibt in Hinblick auf geografische Zuordnungen und Gebietsbezeichnungen in veröffentlichten Karten und Institutsadressen neutral.

\section{Anmerkungen}

1 Es ist allerdings anzumerken, dass Henriette Knörr-Gärtner als Frau in diesen Jahren weitgehend eine Ausnahme darstellte. Wir gendern deshalb nicht, wo nachweislich nur Männer beteiligt waren.

2 Im Zuge dieser Neukonstituierung wurden vielfach auch einzelne, vorbelastete Forscher mitsamt ihrer jeweiligen Forschungsagenda rehabilitiert. Somit finden sich auch zahlreiche Vorläufer für die Forschungen zu Umweltgefahren für das Erbgut bereits in den 1930er Jahren, deren Untersuchung hier allerdings zu weit führen würde (vgl. die genannten Studien von Cottebrune 2008; von Schwerin 2015; Moser 2011; Stoff 2012; sowie Stöckel \& Walter 2002).

3 Tatsächlich stellte sich alsbald heraus, dass Contergan zwar Missbildungen hervorrief, nicht aber die Chromosomen schädigte, sprich teratogen, aber nicht mutagen wirkte. Dem Interesse an Mutagenitätsfragen tat dies allerdings keinen Abbruch.

4 Vgl. zu Breschs Gedanken zur biologischen Zukunft dessen populärwissenschaftlichfuturologisches Buch Zwischenstufe Leben. Evolution ohne Ziel, in dem er die These zu einem „neuen Selbst- und Weltverständnis“ präsentierte, die Evolution des Menschen sei Teil eines „kosmischen Ablaufs“ hin zu einem „planetarischen Riesenwesen“ (Bresch 1977: 66, 251, 312). Ein solch großer Anspruch wurde bereits zuvor etwa vom International Biological Programme (1964-1973) verfolgt, einem richtungsweisenden biologischen Großprojekt, das eine zusammenfassende Sammlung von Beobachtungsdaten („Big Data“) auf globalem Maßstab („Big Science“) zur Sicherung von Gesundheit und Wohlstand erstmals vornahm (vgl. dazu Lindee 2014).

$5 \mathrm{Zu}$ der Vorstellung von Reparaturprozessen auf molekularbiologischer Ebene siehe Schwerin (2008: 202-206); mit Verweis auf Morange (1998: 237); von Schwerin (2015); sowie Yi (2007: 35-72). Ebenso strittig war in der Mutagenitätsforschung die Frage, ob Basenänderungen oder Chromosomen-Aberrationen von größerer Bedeutung wären, ob nur Keimzellen zu untersuchen oder auch somatische Mutationen einzubeziehen waren, oder welche Rolle der Stoffwechsel von Organismen für die Entstehung von Mutationen habe (vgl. DFG 1975: 6).

6 Bresch/Neumann an Klofat, 25.11.1971, Bundesarchiv Koblenz (BArchK), DFG-Akten, Bestand der Kommission für Mutagenitätsfragen, B227/225090.

7 Bresch/Neumann an Klofat, 25.11.1971, BArchK B227/225090.

8 Vogel an Klofat, 19.01.1972, BArchK B227/205590.

9 Klofat an Vogel. 12.09.1973, BArchK B227/225090; vgl. Cottebrune 2012.

10 Protokoll vom 03./04.12.1971, BarchK B227/225090.

11 Bresch an Klofat, 21.12.1971, BArchK B227/225090.

12 Klofat an Schiel, 13.01.1972, BArchK B227/225090.

13 Vgl. die Tagesordnung in: Klofat an Schiel, 13.01.1972, BArchK B227/225090.

14 O.A., Musterinstitut, o.D., BArchK B227/225090.

15 Bresch/Neumann an Klofat, 25.11.1971, BArchK B227/225090.

16 O.A., Musterinstitut, o.D., BArchK B227/225090.

17 Programm der Jahrestagung, o.D., BarchK B 227/225090.

18 Carsten Bresch, Fragebogen an die Teilnehmer, o.D., BArchK B227/225090.

19 Notizen zu Gesprächen betr. Fragebogen, 13.05.72, BarchK B 227/225090.

20 Anwesend waren: Horst Bickel, Carsten Bresch, Karl-Heinz Degenhardt, Heinz-Werner Goedde, Karl Knörr, Friedrich Vogel, Wolf und Peter Starlinger, und Hansjürgen Staudinger. Helmut Baitsch und Widukind Lenz waren verhindert. 
21 Klofat an Mitglieder der Besprechungsgruppe, 10.10.1972, BarchK B227/225090.

22 Ergebnisprotokoll der Besprechung vom 17.11.1972 in Frankfurt, BArchK B227/225090.

23 Protokoll über die Senatssitzung am 06.12.1972, BArchK B227/225090.

24 Das DFG-Programm war nicht die einzige Geldquelle, aus der der Aufbau pränataldiagnostischer Labore und genetischer Beratungsstellen finanziert wurde. So wurden die Beratungsstellen in Marburg und Frankfurt durch „Modellversuche“ des Ministeriums für Jugend, Familie und Gesundheit und der VW-Stiftung zwischen 1972 und 1977 gefördert (Bundesministerium 1979), die Beratungsstelle in München wurde 1973 durch eine an die Stiftung Lebenshilfe gegebene Spende von 350.000 DM aufgebaut und das DFG-Programm erhielt über den Stifterverband für die Deutsche Wissenschaft einen Startzuschuss von der Zementindustrie (!) in Höhe von einer halben Million DM (vgl. Nippert 1991: 55, 57).

25 Bresch an Klofat,05.04.1973, BarchK B227/225092.

26 Wir danken der oder dem anonymen Gutachter/in für diese treffende Formulierung.

\section{Literatur}

Aronova, Elena, Karen S. Baker und Naomi Oreskes 2010. Big Science and Big Data in Biology. From the International Geophysical Year through the International Biological Program to the Long Term Ecological Research (LTER) Network, 1957-Present. Historical Studies in the Natural Sciences (40/2): 183-224.

Bächi, Beat 2010. Zur Krise der westdeutschen Grenzwertpolitik in den 1970er Jahren: Die Verwandlung des Berufskrebses von einem toxikologischen in ein sozioökonomisches Problem. Berichte zur Wissenschaftsgeschichte (33): 419-435.

Baitsch, Helmut 1958. Welche eugenischen Maßnahmen haben heute noch Sinn? Die Heilkunst (6): 213-222.

Behren, Dirk von 2004. Die Geschichte des \$ 218 StGB (Rothenburger Gespräche zur Strafrechtsgeschichte 4). Tübingen: Edition Diskord.

Berridge, Virginia 2007. Marketing Health. Smoking and the Discourse of Public Health in Britain, 1945-2000. Oxford: Oxford University Press.

Bösl, Elsbeth (2009): Politiken der Normalisierung. Zur Geschichte der Behindertenpolitik in der Bundesrepublik Deutschland. Bielefeld: Transcript.

Bresch, Carsten 1964. Klassische und molekulare Genetik. Berlin: Springer.

Bresch, Carsten 1977. Zwischenstufe Leben. Evolution ohne Ziel? München: Piper.

Bundesministerium für Jugend, Familie und Gesundheit (Hg.) 1979. Genetische Beratung. Ein Modellversuch der Bundesregierung in Frankfurt und Marburg. Bonn-Bad Godesberg.

Cottebrune, Anne 2008. Der planbare Mensch. Die Deutsche Forschungsgemeinschaft und die menschliche Vererbungswissenschaft, 1920-1970 (Studien zur Geschichte der Deutschen Forschungsgemeinschaft 2). Stuttgart: Franz Steiner.

Cottebrune, Anne 2012. Die westdeutsche Humangenetik auf dem Weg zu ihrer universitären Institutionalisierung nach 1945 - zwischen Neuausrichtung und Kontinuität. In: Dies. und Wolfgang U. Eckart (Hg.) Das Heidelberger Institut für Humangenetik: Vorgeschichte und Ausbau (1962-2012). Festschrift zum 50jährigen Jubiläum. Heidelberg: Institut für Humangenetik: 28-55.

Cottebrune, Anne 2015. „Reproduktionsentscheidungen dürfen nicht dem Zufall überlassen werden“ Zu Konstruktion und Umgang mit genetischen Risiken im Kontext des Aufbaus humangenetischer Beratungsdienste in der Bundesrepublik der 1970er Jahre. In: Thomas Etzemüller (Hg.). Vom „Volk“ zur „Population“. Interventionistische Bevölkerungspolitik in der Nachkriegszeit. Münster: Westfälisches Dampfboot: 197-222.

De Chadarevian, Soraya 2018. Whose Turn? Chromosome Research and the Study of the Human Genome. Journal of the History of Biology (51/4): 531-55. 
Deutsche Forschungsgemeinschaft 1964. Bericht der Deutschen Forschungsgemeinschaft über ihre Tätigkeit vom 1. Januar bis zum 31. Dezember 1963, Bonn-Bad Godesberg: DFG.

Deutsche Forschungsgemeinschaft 1965. Bericht der Deutschen Forschungsgemeinschaft über ihre Tätigkeit vom 1. Januar bis zum 31. Dezember 1964, Bonn-Bad Godesberg: DFG.

Deutsche Forschungsgemeinschaft 1966. Bericht der Deutschen Forschungsgemeinschaft über ihre Tätigkeit vom 1. Januar bis zum 31. Dezember 1965, Bonn-Bad Godesberg: DFG.

Deutsche Forschungsgemeinschaft 1970. Mitteilungen 3/70. Bonn-Bad Godesberg: DFG.

Deutsche Forschungsgemeinschaft 1971a. Umweltforschung. Aufgaben und Aktivitäten der DFG 1950 bis 1970. Bonn-Bad Godesberg: DFG.

Deutsche Forschungsgemeinschaft 1971b. Mutagenitätsprüfung. (Kommission für Mutagenitätsfragen. Mitteilung 2). Boppard: Boldt.

Deutsche Forschungsgemeinschaft 1971c. Mitteilungen 3/71. Bonn-Bad Godesberg: DFG.

Deutsche Forschungsgemeinschaft 1972a. Mitteilungen 1/72. Bonn-Bad Godesberg: DFG.

Deutsche Forschungsgemeinschaft 1972b. Tätigkeitsbericht der DFG 1971. Bonn-Bad Godesberg: DFG.

Deutsche Forschungsgemeinschaft 1973. Tätigkeitsbericht der DFG 1972. Bonn-Bad Godesberg: DFG.

Deutsche Forschungsgemeinschaft 1974. Tätigkeitsbericht der DFG 1973. Bonn-Bad Godesberg: DFG.

Deutsche Forschungsgemeinschaft 1975. Mutagenitätsprüfung. (Kommission für Mutagenitätsfragen. Mitteilung 3). Boppard: Boldt.

Deutsche Forschungsgemeinschaft 1977a. Schwangerschaftsverlauf und Kindesentwicklung. Bisherige Ergebnisse eines seit 1964 geförderten Schwerpunktprogramms (Stand Mai 1976). Boppard: Boldt.

Deutsche Forschungsgemeinschaft 1977b. Tätigkeitsbericht der DFG 1976. Bonn-Bad Godesberg: DFG.

Deutsche Forschungsgemeinschaft 1977c. Conference on Population Monitoring Methods for Detecting Increased Mutation Rates. Jahreskonferenz 1975. Kommission für Mutagenitätsfragen. Mitteilung 4. Bonn-Bad Godesberg: DFG.

Engels, Jens Ivo 2006. Naturpolitik in der Bundesrepublik. Ideenwelt und politische Verhaltensstile in Naturschutz und Umweltbewegung 1950-1980, Paderborn: Ferdinand Schöningh.

Etzemüller, Thomas 2007: Ein ewigwährender Untergang. Der apokalyptische Bevölkerungsdiskurs im 20. Jahrhundert. Bielefeld: Transcript.

Fünfzehntes Strafrechtsänderungsgesetz. 18. Mai 1976, in: Bundesgesetzblatt 56: 1213-1215.

Gassert, Philipp 2013. Die Entstehung eines neuen Umweltbewusstseins. In: Bernd Greiner, Tim B. Müller und Klaas Voß (Hg.). Erbe des Kalten Krieges (Studien zum Kalten Krieg 6). Hamburg: Hamburger Edition: 343-363.

Hähner-Rombach, Sylvelyn (Hg.) 2015. Geschichte der Prävention: Akteure Praktiken Instrumente. Stuttgart: Steiner.

Harper, Peter S. und Heike I. Petermann 2017. The International Workshops on Genetics, Medicine and History: An Overview, 2003-2015. In: Heike I. Petermann, Peter S. Harper und Susanne Doetz (Hg.). History of Human Genetics. Aspects of its Development and Global Perspectives. Cham: Springer International: 7-38.

Hünemörder, Kai F. 2004. Die Frühgeschichte der globalen Umweltkrise und die Formierung der deutschen Umweltpolitik (1950-1973) (Historische Mitteilungen, Beiheft 53). Stuttgart: Steiner.

Hünemörder, Kai F. 2005. 1972 - Epochenschwelle der Umweltgeschichte? In: Franz-Josef Brüggemeier und Jens Ivo Engels (Hg.). Natur- und Umweltschutz nach 1945. Konzepte, Konflikte, Kompetenzen. Frankfurt a. M.: Campus: 124-144.

Kaldewey, David und Désirée Schauz (Hg.) 2018. Basic and Applied Research. The Language of Science Policy in the Twentieth Century. New York: Berghahn Books.

Knörr, Karl 1971. Möglichkeiten und Konsequenzen der pränatalen Diagnostik kongenitaler Anomalien. Geburtshilfe und Frauenheilkunde (31): 614-622. 
Knörr, Karl 1972. Die pränatale Diagnostik aus der Sicht des Geburtshelfers. In: Jan-Diether Murken, (Hg.). Genetische Familienberatung und pränatale Genetik. München: J. F. Lehmanns: 57-67.

Knörr, Karl 1995. Art. Knörr, Karl. In: Gerhard Bettendorf (Hg.). Zur Geschichte der Endokrinologie und Reproduktionsmedizin. 256 Biographien und Berichte. Berlin, Heidelberg: Springer: 307-312.

Knörr, Karl, Henriette Knörr-Gärtner und Jan Murken 1995. Amniozentese und pränatale Diagnostik. In: Peter Frieß und Peter M. Steiner (Hg.). Forschung und Technik in Deutschland nach 1945, Bonn: Deutsches Museum: 406-407.

Knörr-Gärtner, Henriette 1995. Art. Knörr-Gärtner, Henriette. In: Gerhard Bettendorf (Hg.). Zur Geschichte der Endokrinologie und Reproduktionsmedizin. 256 Biographien und Berichte. Berlin, Heidelberg: Springer: 313-317.

Koller, Siegfried 1972. Die Kooperativ-Studie „Schwangerschaftsverlauf und Kindesentwicklung". In: Erich Saling und Joachim Wolfram Dudenhausen (Hg.). Perinatale Medizin III. 4. Deutscher Kongreß für Perinatale Medizin Berlin, 4.-6. November 1971. Stuttgart: Thieme.

Koller, Siegfried 1983. Risikofaktoren der Schwangerschaft. Auswertung von 7870 Schwangerschaften der prospektiven Untersuchungsreihe „Schwangerschaftsverlauf und Kindesentwicklung" der Deutschen Forschungsgemeinschaft. Berlin und Heidelberg: Springer.

Krige, John 2002. The birth of EMBO and the difficult road to EMBL. Studies in History and Philosophy of Biological E Biomedical Sciences (33): 547-564.

Kröner, Hans-Peter 1997. Von der Eugenik zum genetischen Screening. Zur Geschichte der Humangenetik in Deutschland. In: Franz Petermann (Hg.). Perspektiven der Humangenetik. Medizinische, psychologische und ethische Aspekte. Paderborn: Schöningh: $23-47$.

Labisch, Alfons und Florian Tennstedt 1991. Prävention und Prophylaxe als Handlungsfelder der Gesundheitspolitik in der Frühgeschichte der Bundesrepublik Deutschland (1949-ca. 1965). In: Thomas Elkeles (Hg.). Prävention und Prophylaxe. Theorie und Praxis eines gesundheitspolitischen Grundmotivs in zwei deutschen Staaten 1949-1990. Berlin: Edition Sigma: 129-158.

Leanza, Matthias 2017. Die Zeit der Prävention. Eine Genealogie. Weilerswist: Velbrück Wissenschaft.

Lengwiler, Martin und Stefan Beck 2008. Historizität, Materialität und Hybridität von Wissenspraxen. Die Entwicklung europäischer Präventionsregime im 20. Jahrhundert. Geschichte und Gesellschaft (33): 489-523.

Lindee, Susan 2014. Scaling up. Human genetics as a Cold War network. Studies in History and Philosophy of Biological and Biomedical Sciences (47): 185-190.

Lindner, Ulrike 2007. Die Krise des Wohlfahrtsstaats im Gesundheitssektor. Bundesrepublik Deutschland, Großbritannien und Schweden im Vergleich. Archiv für Sozialgeschichte 47, 2007: 297-324.

Little, Michael A. 2012: Human Population Biology in the Second Half of the Twentieth Century. Current Anthropology (53): 126-138.

Löwy, Ilana 2014a. How genetics came to the unborn. 1960-2000. Studies in History and Philosophy of Biological and Biomedical Sciences (47): 154-162.

Löwy, Ilana 2014b: Prenatal diagnosis: The irresistible rise of the 'visible fetus'. Studies in History and Philosophy of Biological and Biomedical Sciences 47 (2014): 290-299.

Löwy, Ilana 2017. Imperfect Pregnancies. A History of Birth Defects and Prenatal Diagnosis. Baltimore: John Hopkins University Press.

Maasen, Sabine, Maik Tändler, Pascal Eitler und Jens Elberfeld (Hg.) 2011: Das beratene Selbst. Zur Genealogie der Therapeutisierung in den, langen'Siebzigern. Bielefeld: Transcript.

Madarász, Jeannette 2010. Prävention chronischer Herzkreislauf-Krankheiten. BRD, DDR und Großbritannien im Vergleich, 1945-1990. Prävention und Gesundheitsförderung (5/4): 313-318.

Materialienband zum Umweltprogramm der Bundesregierung 1971. Zu Bundestagsdrucksache VI/2710

Moser, Gabriele 2011. Deutsche Forschungsgemeinschaft und Krebsforschung 1920-1970 (Studien zur Geschichte der Deutschen Forschungsgemeinschaft 7). Stuttgart: Steiner. 
Murken, Jan-Diether (Hg.) 1972a. Genetische Familienberatung und pränatale Genetik. München: J. F. Lehmanns.

Murken, Jan-Diether 1972b. Beratungssituation und Fragestellung der pränatalen Genetik. In: Ders. (Hg.): Genetische Familienberatung und pränatale Genetik. München: J. F. Lehmanns: 7-13.

Murken, Jan-Diether 1978. Genetische Beratung und pränatale Diagnostik. Deutsches Ärzteblatt (23): 1379-1396.

Murken, Jan-Diether und Angelika Albert 1978. Möglichkeiten und Probleme der pränatalen genetischen Diagnostik in der Bundesrepublik Deutschland. In: Jan-Diether Murken und Sabine Stengel-Rutkowski (Hg.). Pränatale Diagnostik. Stuttgart: Enke: 1-7.

Murken, Jan-Diether und Sabine Stengel-Rutkowski 1973. Erstes Informationsblatt über das Schwerpunktprogramm „Pränatale Diagnostik genetisch bedingter Defekte. München.

Murken, Jan-Diether und Sabine Stengel-Rutkowski 1974. Zweites Informationsblatt über das Schwerpunktprogramm „Pränatale Diagnostik genetisch bedingter Defekte“. München.

Murken, Jan-Diether, Sabine Stengel-Rutkowski und Eberhard Schwinger (Hg.) 1979. Prenatal Diagnosis. Proceedings of the $3^{\text {rd }}$ European Conference on Prenatal Diagnosis of Genetic Disorders. Stuttart: Enke.

Murken, Jan-Diether und Sabine Stengel-Rutkowski 1973-1982. Informationsblätter über die Dokumentation der Untersuchungen im Rahmen des Schwerpunktprogramms „Pränatale Diagnostik genetisch bedingter Defekte" der Deutschen Forschungsgemeinschaft. Nr. 1 (1973)-Nr. 16 (1982), München.

Mutterschafts-Richtlinien. 16. Dezember 1974 (Inkrafttreten: 1. April 1975). Richtlinien des Bundesausschusses der Ärzte und Krankenkassen über die ärztliche Betreuung während der Schwangerschaft und nach der Entbindung, in: Bundesanzeiger 59, Beilage 12/ 75: 317.

Nemec, Birgit und Gabriele Moser 2017. Counselling, Risk and Prevention in Human Genetic Early Diagnosis in the Federal Republic of Germany. In: Heike I. Petermann, Peter S. Harper und Susanne Doetz (Hg.). History of Human Genetics. Aspects of its Development and Global Perspectives. Cham: Springer International: 367-392.

Nippert, Irmgard 1991. History of Prenatal Genetic Diagnosis in the Federal Republic of Germany. In: Margaret Reid (Hg.). The Diffusion of Four Prenatal Screening Tests across Europe. London: King's Fund Centre: 49-69.

Orth, Karin 2011. Autonomie und Planung der Forschung. Förderpolitische Strategien der Deutschen Forschungsgemeinschaft 1949-1968 (Studien zur Geschichte der Deutschen Forschungsgemeinschaft 8), Stuttgart: Steiner.

Osten, Philipp 2010. Horst Bickel (1918-2000) und der Weg zur Therapie der Phenylketonurie. In: Georg F. Hoffmann, Wolfgang U. Eckart und Philipp Osten (Hg.). Entwicklungen und Perspektiven der Kinder- und Jugendmedizin. 150 Jahre Pädiatrie in Heidelberg. Mainz: Kirchheim: 139-167.

Osten, Philipp 2012. „Wir hatten die besseren Bilder“. Historische, mediale und ethische Aspekte der Zytogenetik. In: Anne Cottebrune und Wolfgang U. Eckart (Hg.). Das Heidelberger Institut für Humangenetik: Vorgeschichte und Ausbau (1962-2012). Festschrift zum 50jährigen Jubiläum. Heidelberg: Institut für Humangenetik: 149-169.

Radkau, Joachim 2011. Die Ära der Ökologie. Eine Weltgeschichte. München: C. H. Beck.

Rosenbrock, Rolf 1998. Die Umsetzung der Ottawa Charta in Deutschland: Prävention und Gesundheitsförderung im gesellschaftlichen Umgang mit Gesundheit und Krankheit. WZB Discussion Paper: 98-201.

Schenk Britta-Marie 2013. Behinderung - Genetik - Vorsorge: Sterilisationspraxis und humangenetische Beratung in der Bundesrepublik. Zeithistorische Forschungen (10/3): 433-545.

Schenk, Britta-Marie, Malte Thießen und Jan-Holger Kirsch (Hg.) 2013. Zeitgeschichte der Vorsorge. Zeithistorische Forschungen (10/3). https://zeithistorische-forschungen.de/ 3-2013. Zugegriffen: 01.02.2019.

Schroeder-Kurth, Traute 1989. Versorgung der Bevölkerung mit humangenetischen Leistungen: Beratung und Diagnostik. In: Dies. (Hg.). Medizinische Genetik in der Bundesrepublik. Frankfurt am Main: Schweitzer: 19-50. 
Schwerin, Alexander von 2008. Der gefährdete Organismus. Biologie und Regierung der Gefahren am Übergang vom „Atomzeitalter“ zur Umweltpolitik (1950-1970). In: Florence Vienne und Christina Brandt (Hg.). Wissensobjekt Mensch. Humanwissenschaftliche Praktiken im 20. Jahrhundert. Berlin: Kadmos: 187-214.

Schwerin, Alexander von 2009. 1961. Die Contergan-Bombe. Der Arzneimittelskandal und die neue risikoepistemische Ordnung der Massenkonsumgesellschaft. In: Nicholas Eschenbruch, Viola Balz, Ulrike Klöppel und Marion Hulverscheidt (Hg.). Arzneimittel des 20. Jahrhunderts. Historische Skizzen von Lebertran bis Contergan. Bielefeld: Transcript: $255-282$.

Schwerin, Alexander von 2010a. Low Dose Intoxication and a Crisis of Regulatory Models. Chemical Mutagens in the Deutsche Forschungsgemeinschaft (DFG), 1963-1973. Berichte zur Wissenschaftsgeschichte (33): 401-418.

Schwerin, Alexander von 2010b. Die Deutsche Atomkommission. Eine biopolitische Institution und die Naturalisierung der Risikopolitik. In: Axel Hüntelmann und Michael Schneider (Hg.). Jenseits von Humboldt. Frankfurt a. M.: Peter Lang: 103-116.

Schwerin, Alexander von 2012a. Humangenetik im Atomzeitalter. Von der Mutationsforschung zum genetischen Bevölkerungsregister. In: Anne Cottebrune und Wolfgang Eckart (Hg.). Das Heidelberger Institut für Humangenetik. Vorgeschichte und Ausbau (1962-2012). Festschrift zum 50jährigen Jubiläum. Heidelberg: Institut für Humangenetik: $82-105$.

Schwerin, Alexander von 2012b. Mutagene Umweltstoffe. Gunter Röhrborn und eine vermeintlich neue eugenische Bedrohung. In: Anne Cottebrune und Wolfgang Eckart (Hg.). Das Heidelberger Institut für Humangenetik. Vorgeschichte und Ausbau (19622012). Festschrift zum 50jährigen Jubiläum. Heidelberg: Institut für Humangenetik: 106-129.

Schwerin, Alexander von 2014. Vom Gift im Essen zu chronischen Umweltgefahren. Lebensmittelzusatzstoffe und die risikopolitische Institutionalisierung der Toxikogenetik in der Bundesrepublik, 1955-1964. Technikgeschichte (81/3): 251-274.

Schwerin, Alexander von 2015. Strahlenforschung. Bio- und Risikopolitik der DFG 1920-1970 (Studien zur Geschichte der Deutschen Forschungsgemeinschaft 10). Stuttgart: Steiner.

Stengel-Rutkowski, Sabine, Brigitte Linse, Roland Brandmaier und Heike Hansen-Kube 1992. Abschlussbericht über die Untersuchungen im Rahmen des Schwerpunktprogramms „Pränatale Diagnose genetisch bedingter Defekte“ der Deutschen Forschungsgemeinschaft (DFG). München.

Stöckel, Sigrid und Ulla Walter (Hg.) 2002. Prävention im 20. Jahrhundert. Historische Grundlagen und aktuelle Entwicklungen in Deutschland. Weinheim und München: Juventus.

Stockhausen, Josef (Hg.) 1971. Programmierte Krankheitsfrüherkennung. Vorsorgeuntersuchungen in der Krankenversicherung. Köln: Deutscher Ärzte Verlag.

Stoff, Heiko 2009. Hexa-Sabbat. Fremdstoffe und Vitalstoffe, Experten und der kritische Verbraucher in der BRD der 1950er und 1960er Jahre. NTM. Zeitschrift für Geschichte der Wissenschaften, Technik und Medizin (17/1): 55-83.

Stoff, Heiko 2012. Wirkstoffe. Eine Wissenschaftsgeschichte der Hormone, Vitamine und Enzyme. 1920-1970 (Studien zur Geschichte der Deutschen Forschungsgemeinschaft 9). Stuttgart: Steiner.

Stoff, Heiko 2015. Gift in der Nahrung. Zur Genese der Verbraucherpolitik in Deutschland Mitte des 20. Jahrhunderts. Stuttgart: Steiner.

Thomaschke, Dirk 2014: In der Gesellschaft der Gene. Räume und Subjekte der Humangenetik in Deutschland und Dänemark, 1950-1990. Bielefeld: Transcipt.

Uekötter, Frank 2003. Von der Rauchplage zur ökologischen Revolution. Eine Geschichte der Luftverschmutzung in Deutschland und den USA 1880-1970 (Veröffentlichungen des Instituts für soziale Bewegungen A/26). Essen: Klartext.

Umweltprogramm der Bundesregierung 1971. Bundestagsdrucksache VI/2710

Unbekannt ["Mst"] 1976. Diagnostik genetischer Störungen durch Amniozentese. Deutsches Ärzteblatt (11): 724 .

Unbekannt [„yx“] 1966. Chromosomen-Untersuchungen fötaler Zellen. Deutsches Ärzteblatt (51): 2964. 
Vogel, Friedrich und Walter Fuhrmann 1968. Genetische Familienberatung. Ein Leitfaden für den Arzt. Berlin, Heidelberg: Springer.

Waldschmidt, Anne 1996. Das Subjekt in der Humangenetik. Expertendiskurse zu Programmatik und Konzeption der genetischen Beratung 1945-1990. Münster: Westfälisches Dampfboot.

Weingart, Peter, Jürgen Kroll und Kurt Bayertz 1988. Rasse, Blut und Gene. Geschichte der Eugenik und Rassenhygiene in Deutschland. Frankfurt a. M.: Suhrkamp.

Wenkel, Simone und Ute Deichmann (Hg.) 2007. Max Delbrück and Cologne. An Early Chapter of German Molecular Biology, New Jersey, London et al.: World Scientific.

Yi, Doogab 2007. The coming of reversibility: The discovery of DNA repair between the atomic age and the information age. Historical Studies in the Physical and Biological Sciences 37: 35-72.

Birgit Nemec

Institut für Geschichte und Ethik der Medizin

Ruprecht-Karls-Universität Heidelberg

Im Neuenheimer Feld 327

69120 Heidelberg

Deutschland

birgit.nemec@histmed.uni-heidelberg.de

Fabian Zimmer

Rachel Carson Center for Environment and Society

LMU München

Leopoldstraße 11a

80802 München

Deutschland

fabian.zimmer@rcc.lmu.de 